\title{
Sodium Butyrate Ameliorates Oxidative Stress-Induced Intestinal Epithelium Barrier Injury and Mitochondrial Damage through AMPK-Mitophagy Pathway
}

\author{
Xin Li $\mathbb{D}^{1,2,3,4}$ Chunchun Wang, ${ }^{1,2,3,4}$ Jiang Zhu, ${ }^{1,2,3,4}$ Qian Lin, ${ }^{1,2,3,4}$ Minjie Yu, ${ }^{1,2,3,4}$ \\ Jiashu Wen, ${ }^{1,2,3,4}$ Jie Feng, ${ }^{1,2,3,4}$ and Caihong $\mathrm{Hu} \mathbb{D}^{1,2,3,4}$ \\ ${ }^{1}$ College of Animal Science, Zhejiang University, Hangzhou 310058, China \\ ${ }^{2}$ Key Laboratory of Molecular Animal Nutrition (Zhejiang University), Ministry of Education, China \\ ${ }^{3}$ Key Laboratory of Animal Nutrition and Feed Science (Eastern of China), Ministry of Agriculture and Rural Affairs, China \\ ${ }^{4}$ Key Laboratory of Animal Feed and Nutrition of Zhejiang Province, China
}

Correspondence should be addressed to Caihong Hu; chhu@zju.edu.cn

Received 9 November 2021; Revised 3 January 2022; Accepted 12 January 2022; Published 29 January 2022

Academic Editor: Hao Wu

Copyright (C) 2022 Xin Li et al. This is an open access article distributed under the Creative Commons Attribution License, which permits unrestricted use, distribution, and reproduction in any medium, provided the original work is properly cited.

\begin{abstract}
Sodium butyrate has gained increasing attention for its vast beneficial effects. However, whether sodium butyrate could alleviate oxidative stress-induced intestinal dysfunction and mitochondrial damage of piglets and its underlying mechanism remains unclear. The present study used a hydrogen peroxide- $\left(\mathrm{H}_{2} \mathrm{O}_{2^{-}}\right)$induced oxidative stress model to study whether sodium butyrate could alleviate oxidative stress, intestinal epithelium injury, and mitochondrial dysfunction of porcine intestinal epithelial cells (IPEC-J2) in AMPK-mitophagy-dependent pathway. The results indicated that sodium butyrate alleviated the $\mathrm{H}_{2} \mathrm{O}_{2}$-induced oxidative stress, decreased the level of reactive oxygen species (ROS), increased mitochondrial membrane potential (MMP), mitochondrial DNA (mtDNA), and mRNA expression of genes related to mitochondrial function, and inhibited the release of mitochondrial cytochrome $\mathrm{c}(\mathrm{Cyt} \mathrm{c})$. Sodium butyrate reduced the protein expression of recombinant NLR family, pyrin domain-containing protein 3 (NLRP3) and fluorescein isothiocyanate dextran $4 \mathrm{kDa}$ (FD4) permeability and increased transepithelial resistance (TER) and the protein expression of tight junction. Sodium butyrate increased the expression of light-chain-associated protein B (LC3B) and Beclin-1, reduced the expression of P62, and enhanced mitophagy. However, the use of AMPK inhibitor or mitophagy inhibitor weakened the protective effect of sodium butyrate on mitochondrial function and intestinal epithelium barrier function and suppressed the induction effect of sodium butyrate on mitophagy. In addition, we also found that after interference with AMPK $\alpha$, the protective effect of sodium butyrate on IPEC-J2 cells treated with $\mathrm{H}_{2} \mathrm{O}_{2}$ was suppressed, indicating that AMPK $\alpha$ is necessary for sodium butyrate to exert its protective effect. In summary, these results revealed that sodium butyrate induced mitophagy by activating AMPK, thereby alleviating oxidative stress, intestinal epithelium barrier injury, and mitochondrial dysfunction induced by $\mathrm{H}_{2} \mathrm{O}_{2}$.
\end{abstract}

\section{Introduction}

The intestinal epithelium barrier is known as the important barrier to prevent the invasion of toxins or antigens [1]. Studies have found that oxidative stress is involved in the intestinal barrier impairment of piglets, which mainly refers to the imbalance of reactive oxygen species (ROS) and antioxidant system $[2,3]$. Under oxidative stress, the overproduction of ROS can cause lipid peroxidation, protein, and
DNA damage [4]. Multiple studies have shown that dietary sodium butyrate can protect the intestinal barrier of piglets by providing energy for intestinal epithelial cells, antiinflammation, histone deacetylation, immune regulation [5-7], etc. The in vivo rat experiments and in vitro intestinal epithelial cell experiments have demonstrated that the protective effects of intestinal barrier function by sodium butyrate are related to its antioxidant capacity [8-10]. Since mitochondria are the main resource of ROS, the antioxidant 
effect of sodium butyrate may result from the targeting on mitochondria.

The gut needs a lot of energy to maintain homeostasis, which is mainly supplied by intracellular mitochondria [9, 10]. However, under oxidative stress, mitochondria are not only the main resource of ROS but also an important attack target of it [11]. Impaired mitochondria induced by oxidative stress can produce more than ten times more ROS than the normal mitochondria, which further aggravated mitochondrial damage [12]. In order to block this vicious cycle, the body will selectively remove damaged mitochondria via lysosome degradation, a process called mitophagy [13]. A recent study reported that sodium butyrate can induce mitophagy in Chinese hamster ovary cells [14]. We speculate that the antioxidant effect of sodium butyrate is related to decreasing mitochondrial ROS production and mitophagy.

Adenosine monophosphate-activated protein kinase (AMPK) is a key energy sensor, as well as an important oxidative stress sensor, which exerts an important role in mitochondrial function and mitophagy $[15,16]$. AMPK $\alpha$ is the catalytic core of AMPK, and Toyama et al. found that AMPK is activated in cells under energy-deficient situation, as indicated by that AMPK promoted the further recruitment of multiple proteins related to mitochondrial division by phosphorylating the downstream protein mitochondrial fission factor (MFF), thereby causing mitochondrial division $[17,18]$, which links the energy receptor AMPK with mitochondrial division. It is well known that sodium butyrate is an important energy substance for intestinal epithelial cells and has the functions of alleviating oxidative stress and protecting the intestinal barrier $[9,19]$. Mollica et al. have reported that feeding sodium butyrate to insulin-resistant obese mice can increase liver AMPK activity, reduce ROS production, and improve liver mitochondrial function [20]. However, whether sodium butyrate can alleviate oxidative stress and improve mitochondrial function through AMPK remains unclear. Further research is needed to study the mechanism through which sodium butyrate alleviates oxidative stress and improves intestinal barrier function.

Therefore, this experiment utilized an IPEC-J2 cell oxidative stress model by $\mathrm{H}_{2} \mathrm{O}_{2}$ to study the effects of sodium butyrate on intestinal barrier injury, mitochondrial function, mitophagy, and the underlying molecular mechanisms.

\section{Material and Methods}

2.1. Chemicals. The IPEC-J2 cell was a courtesy from Prof. Yin Yulong, Institute of Subtropical Agriculture, Chinese Academy of Sciences. $\mathrm{H}_{2} \mathrm{O}_{2}$ (Sinopharm Group, China), DMEM-F12 medium (Shanghai Yuanpei Biotechnology, China), and fluorescein isothiocyanate dextran $4 \mathrm{kDa}$ (FD4) were obtained from Sigma-Aldrich (St. Louis, Missouri, USA). Trypsin (Beyotime Biotechnology, China), phosphate-buffered saline (PBS) (Bozan Biotechnology, China), penicillin-streptomycin (Solabao Biotechnology, China), fetal bovine serum (Gemini, Australia), CCK-8 kit (Beyotime Biotechnology, China), MitoSpy ${ }^{\mathrm{TM}}$ Red CMXRos (Biolegend, USA), immunofluorescence fixative (Sevier Biotechnology, China), Triton-X 100 (Sigma-Aldrich, St. Louis,
MO, USA), Glycine (Sinopharm Group, China), DAPI (Beyotime Biotechnology, China), Goat Anti-Mouse IgG Dylight 594 (Earthox, USA), Goat Anti-Mouse IgG Dylight 488 (Earthox, USA), mitochondrial division inhibitor (Mdivi-1) (Selleck, USA), AMPK inhibitor (Compound C, CC) (Selleck, USA), Lipofectamine RNAiMAX, and Lipofectamine 2000 were obtained from Invitrogen (Invitrogen, USA).

2.2. Experimental Design and Cell Culture. IPEC-J2 cells were cultured in DMEM-F12 complete medium (10\% fetal bovine serum and $1 \%$ antibiotics (penicillin and streptomycin)). The culture conditions are $37^{\circ} \mathrm{C}, 5 \% \mathrm{CO}_{2}$, and $95 \%$ humidity in a carbon dioxide incubator, and the medium is changed every 2 days. The IPEC-J2 cell oxidative stress model is constructed by $600 \mu \mathrm{mol} / \mathrm{L} \mathrm{H}_{2} \mathrm{O}_{2}$ for 8 hours. This study is divided into three experiments. Experiment 1: control group (control), sodium butyrate group $(\mathrm{NaB})$, oxidative stress group $\left(\mathrm{H}_{2} \mathrm{O}_{2}\right)$, and sodium butyrate+oxidative stress group $\left(\mathrm{NaB}+\mathrm{H}_{2} \mathrm{O}_{2}\right) ; 600 \mu \mathrm{mol} / \mathrm{L} \mathrm{H}_{2} \mathrm{O}_{2}$ was treated with cells in the $\mathrm{H}_{2} \mathrm{O}_{2}$ group and $\mathrm{NaB}+\mathrm{H}_{2} \mathrm{O}_{2}$ group for 8 hours. The cells in the control group and $\mathrm{NaB}$ group were added with the same amount of PBS. Experiment 2: control group (control), oxidative stress group $\left(\mathrm{H}_{2} \mathrm{O}_{2}\right)$, sodium butyrate+oxidative stress group $\left(\mathrm{NaB}+\mathrm{H}_{2} \mathrm{O}_{2}\right)$, sodium butyrate+oxidative stress+mitophagy inhibitor group $\left(\mathrm{NaB}+\mathrm{H}_{2} \mathrm{O}_{2}+\right.$ Mdivi-1), and sodium butyrate+oxidative stress+AMPK signal pathway inhibitor group $\left(\mathrm{NaB}+\mathrm{H}_{2} \mathrm{O}_{2}+\right.$ Compound $\left.\mathrm{C}\right) ; 1$ hour before adding $\mathrm{H}_{2} \mathrm{O}_{2}$ or PBS, mitophagy inhibitor (Mdivi-1, $1 \mu \mathrm{mol} / \mathrm{L}$ ) or AMPK signaling pathway inhibitor (Compound $\mathrm{C}, 10 \mu \mathrm{mol} / \mathrm{L}$ ) was added into cells. Experiment 3: the experiment is divided into six groups: control group (control+siAMPK $\alpha$ ), oxidative stress group $\left(\mathrm{H}_{2} \mathrm{O}_{2}\right)$, oxidative stress+interference AMPK $\alpha\left(\mathrm{H}_{2} \mathrm{O}_{2}+\mathrm{siAMPK} \alpha\right)$, sodium butyrate+oxidative stress group $\left(\mathrm{NaB}+\mathrm{H}_{2} \mathrm{O}_{2}\right)$, and sodium butyrate+oxidative stress+interference AMPK $\alpha$ group $(\mathrm{NaB}$ $+\mathrm{H}_{2} \mathrm{O}_{2}+$ siAMPK $\alpha$ ). The cells in control, $\mathrm{H}_{2} \mathrm{O}_{2}$, and $\mathrm{NaB}$ $+\mathrm{H}_{2} \mathrm{O}_{2}$ groups were transfected with siControl. And the cells in control+siAMPK $\alpha, \mathrm{H}_{2} \mathrm{O}_{2}+$ siAMPK $\alpha$, and $\mathrm{NaB}+\mathrm{H}_{2} \mathrm{O}_{2}$ + siAMPK $\alpha$ groups were transfected with siAMPK $\alpha$.

2.3. Cell Viability Experiment. IPEC-J2 cells in logarithmic phase were seeded in a 96-well plate, $10 \mu \mathrm{l}$ of CCK-8 solution was added to each well, incubated at $37^{\circ} \mathrm{C}$ for $4 \mathrm{~h}$, and the absorbance at $450 \mathrm{~nm}$ was measured with a microplate reader (FLx800, Bio-Tek Instruments Inc., Winooski, USA). The cell viability is normalized based on the absorbance of cells in the control group.

2.4. Transepithelial Electrical Resistance (TER) and FD4 Flux of IPEC-J2 Cells in the Transwell System. According to the previous study, transwell (Corning, NT, USA) is premoistened with DMEM-F12 medium in the incubator for more than 30 minutes before use [21]. After trypsinization of the cells, stop the reaction with fresh medium, centrifuge at $1500 \mathrm{rpm}$ for $5 \mathrm{~min}$, discard the solution and add a certain volume of fresh medium, adjust the cell concentration to 1 $\times 10^{6}$ cells $/ \mathrm{ml}$, add $150 \mu \mathrm{l}$ cells to the upper chamber suspension, add $1.5 \mathrm{ml}$ of fresh medium to the lower chamber, 
and place it in a carbon dioxide incubator for culture. Change the fluid every 2 days. According to the previous study, TER was measured by the Millicell-ERS resistance system (Millipore; Bedford, MA) at three different points in each transwell, and the average of the three measured values was actual TER [22]. The TER value measured without inoculation of the cell culture is the blank TER. The cell monolayer TER $=($ measured TER - Blank TER $) \times$ transwell effective membrane area and expressed in $\left(\Omega \cdot \mathrm{cm}^{2}\right)$. FD4 (final concentration $1 \mathrm{mg} / \mathrm{ml}$ ) was added to the upper chamber of transwell, and $50 \mu \mathrm{l}$ (supplement with the same volume of fresh medium after sampling) was sampled in the lower chamber at 24 hours and 48 hours, to determine the concentration of FD4 and calculate the permeability of the intestinal epithelium barrier FD4 by a fluorescence microplate reader (FLx800, Bio-Tek Instruments Inc., Winooski, USA).

2.5. Determination of Cellular Antioxidant Capacity and IL$1 \beta$. After cell treatment, add cell lysate, lyse on ice for $10 \mathrm{~min}$, centrifuge at $4^{\circ} \mathrm{C}$ for $10 \mathrm{~min}(12000 \mathrm{rpm} / \mathrm{min})$, and take the supernatant for testing. According to the ELISA kit instructions (Nanjing Jiancheng Bioengineering Institute, Jiangsu, China), the activity of superoxide dismutase (SOD) and GSH (glutathione reductase) antioxidant enzymes, the MDA (malondialdehyde) content, and IL- $1 \beta$ content were measured for each sample.

2.6. Determination of Cellular ROS Level. Flow cytometry was conducted to determine the cellular ROS level by a ROS-sensitive fluorescence indicator $2^{\prime}, 7^{\prime}$-dichlorofluorescein diacetate (DCFH-DA; Sigma-Aldrich) [23]. In brief, the cells in the $2 \mathrm{ml}$ centrifuge were incubated with $1 \mathrm{ml}$ serum-free diluted DCFH-DA $(1: 1000)$ at $37^{\circ} \mathrm{C}$ for $30 \mathrm{~min}$ in the dark. After washed twice with PBS and resuspended, flow cytometry (BD, USA) was used to detect the cellular ROS. The data was calculated by FlowJo software 10.4 (V 7.6.1).

2.7. Measurement of Mitochondrial Membrane Potential $(M M P)$. The mitochondrial membrane potential was measured by mitochondrial membrane potential assay kit (Beyotime Biotechnology, China) according to the previous study [24]. JC-1 monomers (green) can form aggregates (red fluorescence) in the mitochondria with high $\triangle \Psi \mathrm{m}$, which cannot form aggregates in the mitochondria with low $\triangle \Psi \mathrm{m}$. Briefly, the cells were incubated with JC-1 working solution $37^{\circ} \mathrm{C}$ for 25 minutes. After washed for three times with PBS, flow cytometry (BD, USA) or a confocal laser microscope (FV1000, Olympus, Japan) was used to detect mitochondrial membrane potential (MMP).

2.8. mRNA Expression Level Analysis by Quantitative RealTime PCR (RT-qPCR). The total RNA extraction was conducted by Trizol according to the manufacturer's instruction (Takara, Japan). After detecting RNA concentration and purity, it is used for cDNA synthesis (Vazyme, Nanjing, China) and then for RT-qPCR according to the manufacturer's instructions (qPCR, master mix, Vazyme, Nanjing, China) as previously described [25]. The primer sequences are shown in supplementary Table 1. Glyceraldehyde-3phosphate dehydrogenase (GAPDH) was used as an internal reference gene to calculate the relative expression of each gene, and the data was normalized using the $2^{-\Delta \Delta \mathrm{Ct}}$ method.

2.9. Western Blot Experiment. The western blot experiment was conducted according to the previous study [26]. Total protein extraction and quantification were performed through the RIPA lysis buffer and BCA protein Assay Kit (Beyotime Biotechnology, Shanghai, China). Proteins from the jejunum mucosa were run and isolated through SDSPAGE. Then, proteins were transferred to a polyvinylidene difluoride membrane. After blocking with 5\% defat milk powder at room temperature for one hour, blots were incubated with specific primary antibodies overnight and then with horseradish peroxidase- (HRP-) conjugated secondary antibodies. BeyoECL Moon kit (Beyotime Biotechnology, China) was used to visualize the protein blot. The primary antibodies including $\beta$-actin, PINK1, Parkin, Claudin-1, Occludin, ZO-1, LC3B, Beclin-1, and P62 were purchased from Santa Cruz Biotechnology. Cytochrome c, NLRP3, Caspase-1, IL-1 $\beta$, HRP-conjugated anti-rabbit IgG, and HRP-conjugated anti-mouse IgG antibodies were purchased from HUABIO (Hangzhou, China).

2.10. Ultrastructure of IPEC-J2 Cell Mitochondria. Briefly, the IPEC-J2 cells were fixed in $2.5 \%$ glutaraldehyde at $4^{\circ} \mathrm{C}$ overnight and then postfixed with $1 \%$ OsO4. After dehydration (gradient concentration of ethanol), infiltration (Spurr resin), embedding, ultrathin sectioning (LEICA EM UC7), and staining (uranyl acetate and alkaline lead citrate for 5 to 10 minutes, respectively), the samples were observed by transmission electron microscope (Hitachi Model H-7650, Tokyo, Japan).

2.11. Mitochondria Mark by MitoSpy ${ }^{T M}$ Red CMXRos. MitoSpy ${ }^{\mathrm{TM}}$ Mitochondrial Localization Probe (Biolegend, USA) is a cell-permeable fluorescent chemical reagent used to label mitochondria in living cells. According to the manufacturer's instruction, we centrifuge the lyophilized reagent in the centrifuge tube to ensure that the reagent is at the bottom of the vial, add $94 \mu \mathrm{l}$ DMSO to each tube, and reconstitute MitoSpy ${ }^{\mathrm{TM}}$ Red CMXRos to a concentration of $1 \mathrm{mmol} / \mathrm{L}$ with DMSO. The stock solution is diluted in serum-free medium to make MitoSpy ${ }^{\mathrm{TM}}$ Red CMXRos working solution. After the cell treatment is over, wash once with warm $1 \mathrm{X}$ PBS. Add the diluted MitoSpy ${ }^{\mathrm{TM}}$ Red CMXRos solution to living cells and place them in an incubator at $37^{\circ} \mathrm{C}$ for 20 $30 \mathrm{~min}$. Wash the cells twice with warm 1X PBS or medium. Fix the cells with immunofluorescence fixative (4\% paraformaldehyde) for 10-20 minutes at room temperature. Wash the cells twice with $1 \mathrm{X}$ PBS for subsequent experiments.

2.12. Immunofluorescence Analysis. The IPEC-J2 cells were seeded in a laser confocal culture dish. After the indicated treatment, the cells were washed twice with precooled PBS, and go through the steps of fixation, permeabilization (Tritox X-100, Beyotime Biotechnology, Shanghai, China), blocking, primary antibody incubation (Claudin-1, LC3B, 
and PINK1), secondary antibody incubation (Dylight-conjugated, Earthox, USA), nuclear staining (DAPI), and sealing. The samples were observed and photographed under a confocal laser microscope (FV1000, Olympus, Japan).

2.13. siRNA and Cell Transfection. IPEC-J2 cells were seeded into 6-well plates to grow about $80 \%$ confluent. The next day, individual targeted siRNA and plasmid were mixed with Lipofectamine RNAiMAX or Lipofectamine 2000 (Invitrogen, USA). The siRNA sequence is as follows: siAMPK $\alpha$ ( $5^{\prime}$-GCT GCACCAGAAGTAATTTTT- $\left.3^{\prime}\right)$ and siControl ( $5^{\prime}$-UUCUCCGAACGUGUCACG UTT- $\left.3^{\prime}\right)$. The RNAiMAX/siRNA mixture was added to IPEC-J2 cells in antibiotic-free medium and cultured for 4-6 h. Medium containing siRNA was refreshed with the general medium. Sodium butyrate and $\mathrm{H}_{2} \mathrm{O}_{2}$ were added to the indicated group according to the experimental design. Then, the samples were collected for subsequent experiments.

2.14. Statistical Analysis. The experimental data was statistically analyzed using the SPSS 20.0 software. One-way ANOVA was used for test analysis. Turkey method was used for multiple comparisons, and the GraphPad Prism 7.01 software was used for graphing. $P<0.05$ indicates significant difference.

\section{Results}

3.1. Effect of Sodium Butyrate on the Oxidative Stress of IPEC-J2 Cells Treated by $\mathrm{H}_{2} \mathrm{O}_{2}$. The IPEC-J2 cells were treated with different concentrations of $\mathrm{H}_{2} \mathrm{O}_{2}(200 \mu \mathrm{mol} / \mathrm{L}$, $400 \mu \mathrm{mol} / \mathrm{L}, 600 \mu \mathrm{mol} / \mathrm{L}, 800 \mu \mathrm{mol} / \mathrm{L}$, and $1000 \mu \mathrm{mol} / \mathrm{L}$ ) for 8 hours, and the cell viability was determined. As shown in Figure 1(a), it was found that $\mathrm{H}_{2} \mathrm{O}_{2}$ of 600,800 , and $1000 \mu \mathrm{mol} / \mathrm{L}$ significantly reduced cell viability $(P<0.05)$. Cells were treated with $0.25,0.5,1,2,4,8$, and $16 \mu \mathrm{mol} / \mathrm{L}$ sodium butyrate for 24 hours, and it was found that 0.25 $2 \mathrm{mmol} / \mathrm{L}$ sodium butyrate had no significant effect on cell viability, and more than $4 \mathrm{mmol} / \mathrm{L}$ would significantly inhibit cell growth (Figure 1(b)). In addition, the cells were treated with $0.25,0.5,1,2$, and $4 \mathrm{mmol} / / \mathrm{L}$ sodium butyrate, and it was found that $1 \mathrm{mmol} / \mathrm{L}$ sodium butyrate could effectively inhibit the decrease in cell viability induced by $\mathrm{H}_{2} \mathrm{O}_{2}$ $(P<0.05)$ (Figure $1(c))$. Therefore, the follow-up experiments used $600 \mu \mathrm{mol} / \mathrm{L} \quad \mathrm{H}_{2} \mathrm{O}_{2}$-induced oxidative stress model and $1 \mathrm{mmol} / \mathrm{L}$ sodium butyrate pretreatment. As shown in Figures 1(d)-1(f), compared with the control group, $\mathrm{H}_{2} \mathrm{O}_{2}$ treatment significantly reduced the SOD and GSH activities of IPEC-J2 cells $(P<0.05)$ and increased the MDA level $(P<0.05)$; meanwhile, compared with the $\mathrm{H}_{2} \mathrm{O}_{2}$ group, sodium butyrate significantly inhibited the decreased activity of SOD and GHS induced by $\mathrm{H}_{2} \mathrm{O}_{2}$, alleviated the increase of MDA level $(P<0.05)$. Similarly, flow cytometry data showed that, in comparison with the control group, sodium butyrate pretreatment significantly inhibited the increase in ROS levels induced by $\mathrm{H}_{2} \mathrm{O}_{2} \quad(P<0.05)$ (Figure 1(g)).
3.2. Effect of Sodium Butyrate on the Mitochondrial Function of IPEC-J2 Cells Treated with $\mathrm{H}_{2} \mathrm{O}_{2}$. Flow cytometry data showed that compared with the control group, $\mathrm{H}_{2} \mathrm{O}_{2}$ significantly increased the proportion of depolarized cells $(P<0.05)$, while sodium butyrate pretreatment reversed the increase in the proportion of depolarized cells induced by $\mathrm{H}_{2} \mathrm{O}_{2}(P<0.05)$ (Figure 2(a)). In addition, under laser confocal microscope, we found that compared with the control group, $\mathrm{H}_{2} \mathrm{O}_{2}$ reduced the ratio of JC-1 aggregates to monomer $(P<0.05)$, indicating that the mitochondrial membrane potential was reduced, and the sodium butyrate treatment alleviated the decrease of cell mitochondrial membrane potential induced by $\mathrm{H}_{2} \mathrm{O}_{2}(P<0.05)$ (Figure 2(b)). By detecting the amount of mitochondrial DNA (mtDNA) (Figure 2(c)) and the mRNA expression level of mitochondrial function-related genes (Figure 2(d)), it was found that compared with the control group, $\mathrm{H}_{2} \mathrm{O}_{2}$ treatment significantly reduced the amount of mtDNA and the mRNA expression of mitochondrial function-related gene mitochondrial transcription factor A (TFAM), nuclear respiratory factor-1 (NRF-1), and peroxisomal proliferatoractivated receptor-g coactivator- $1 \alpha \quad(\mathrm{PGC}-1 \alpha) \quad(P<0.05)$; sodium butyrate significantly alleviated the reduction of mtDNA and mRNA level of mitochondrial functionrelated genes induced by $\mathrm{H}_{2} \mathrm{O}_{2}(P<0.05)$. Similarly, under the transmission electron microscope, it was observed (Figure 2(e)) that the cells in the $\mathrm{H}_{2} \mathrm{O}_{2}$ group showed obvious mitochondrial swelling, mitochondrial crista breakage, and mitochondrial vacuolization; sodium butyrate significantly improved the ultrastructure of mitochondria. As shown in Figure 2(f), compared with the control group, $\mathrm{H}_{2} \mathrm{O}_{2}$ increased the level of cytochrome $\mathrm{c}$ (Cyt c) in the cytoplasm $(P<0.05)$ and decreased the level of Cyt $\mathrm{c}$ in the mitochondria $(P<0.05)$, suggesting that $\mathrm{H}_{2} \mathrm{O}_{2}$ increased the permeability of the inner and outer mitochondrial membranes and resulted in the release of mitochondrial Cyt $c$ into the cytoplasm, indicating that the mitochondrial function is damaged; compared with the $\mathrm{H}_{2} \mathrm{O}_{2}$ group, sodium butyrate alleviated the release of mitochondrial Cyt $\mathrm{c}$ caused by $\mathrm{H}_{2} \mathrm{O}_{2}(P<0.05)$, indicating that sodium butyrate treatment can help improve mitochondrial function under oxidative stress.

3.3. Effect of Sodium Butyrate on Inflammasome and Inflammatory Factors of IPEC-J2 Cells Treated with $\mathrm{H}_{2} \mathrm{O}_{2}$. As shown in Figures 3(a)-3(c), compared with the control group, $\mathrm{H}_{2} \mathrm{O}_{2}$ significantly increased the mRNA level and protein level of NLRP3, Caspase-1, and IL- $1 \beta$ in IPEC-J2 cells $(P<0.05)$ and increased the content of IL- $1 \beta$ $(P<0.05)$; compared with the $\mathrm{H}_{2} \mathrm{O}_{2}$ group, sodium butyrate significantly reduced the mRNA level and protein expression of NLRP3, Caspase- 1 , and IL- $1 \beta(P<0.05)$ and reduced the content of IL-1 $\beta(P<0.05)$.

3.4. Effect of Sodium Butyrate on the Intestinal Epithelium Barrier Function of IPEC-J2 Cells Treated with $\mathrm{H}_{2} \mathrm{O}_{2}$. As shown in Figure 4(a), compared with the control group, $\mathrm{H}_{2} \mathrm{O}_{2}$ significantly reduced TER $(P<0.05)$ and increased the flux of FD4 $(P<0.05)$. Compared with the $\mathrm{H}_{2} \mathrm{O}_{2}$ 


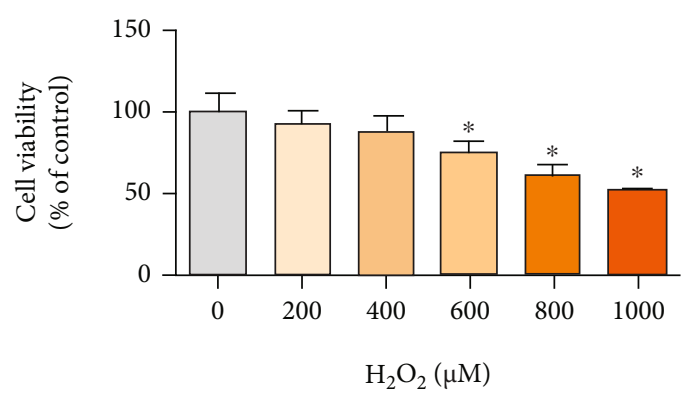

(a)

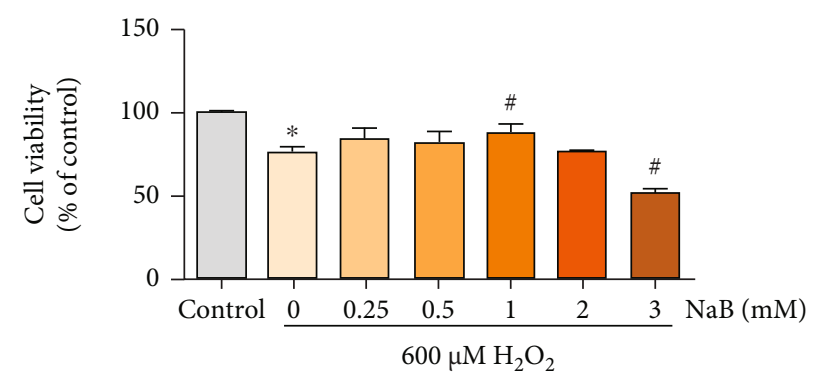

(c)

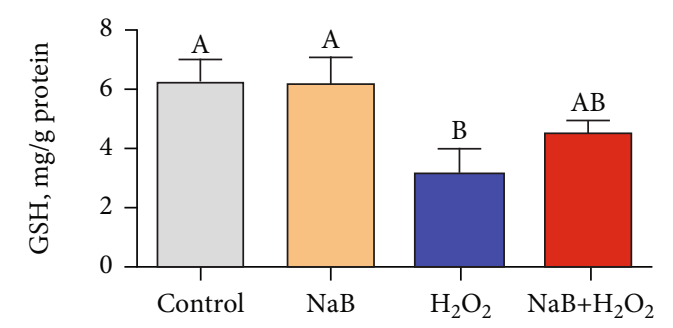

(e)

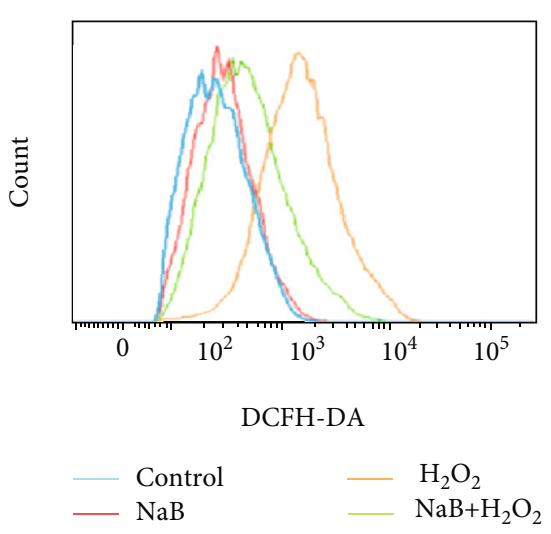

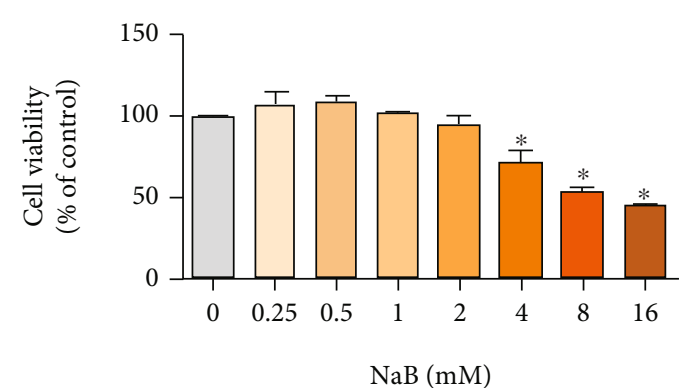

(b)

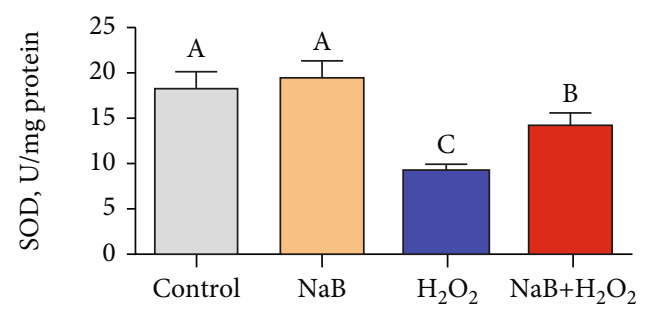

(d)

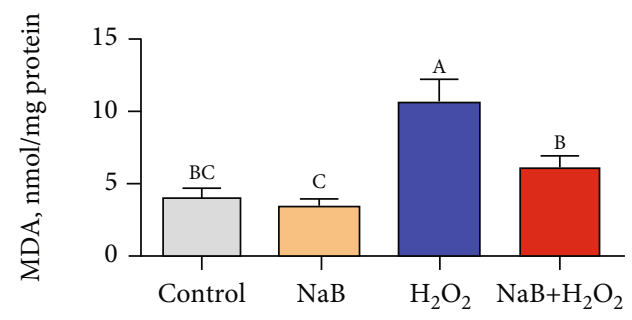

(f)

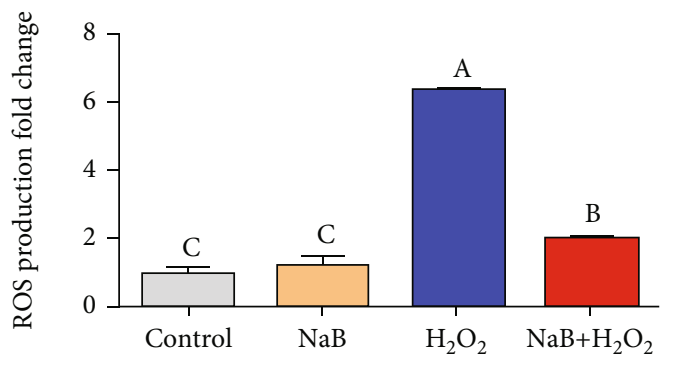

(g)

Figure 1: Effects of sodium butyrate $(\mathrm{NaB})$ on the oxidative stress of porcine intestinal epithelial cells (IPEC-J2) treated by hydrogen peroxide (H2O2). (a-c) The effect of different concentrations of $\mathrm{H} 2 \mathrm{O} 2$ and $\mathrm{NaB}$ on cell viability. (d-f) Cell superoxide dismutase (SOD), glutathione reductase (GSH) activity, and malondialdehyde (MDA) level. (g) Flow cytometry to quantify the reactive oxygen species (ROS) level of IPEC-J2 cells. Different superscript letters within the same row mean significant difference $(P<0.05)$. $*$ and \# mean significant differences $(P<0.05)$ with the control group.

group, sodium butyrate pretreatment significantly alleviated the $\mathrm{H}_{2} \mathrm{O}_{2}$-induced decrease in TER and the increase in FD4 flux $(P<0.05)$. In comparison with the control group, $\mathrm{H}_{2} \mathrm{O}_{2}$ significantly reduced the expression of Claudin-1, Occludin, and ZO-1 of IPEC-J2 cells $(P<0.05)$; compared with the $\mathrm{H}_{2} \mathrm{O}_{2}$ group, sodium butyrate pretreatment significantly increased the protein expression of Claudin-1, Occludin, and ZO-1 in IPEC-J2 cells $(P<0.05$ ) (Figure 4(b)). As shown in Figure 4(c), the Claudin-1 protein of IPEC-J2 cells in the control group is mainly 

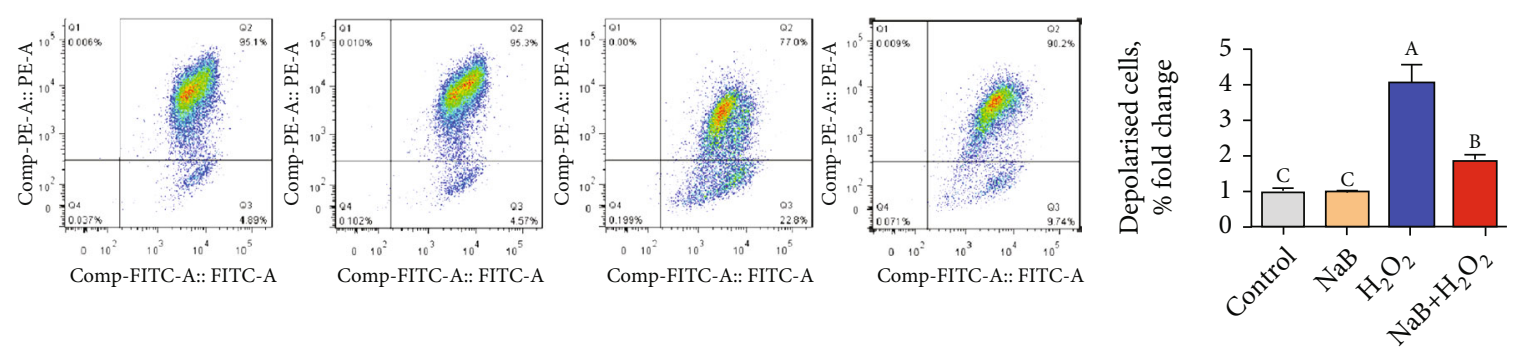

(a)
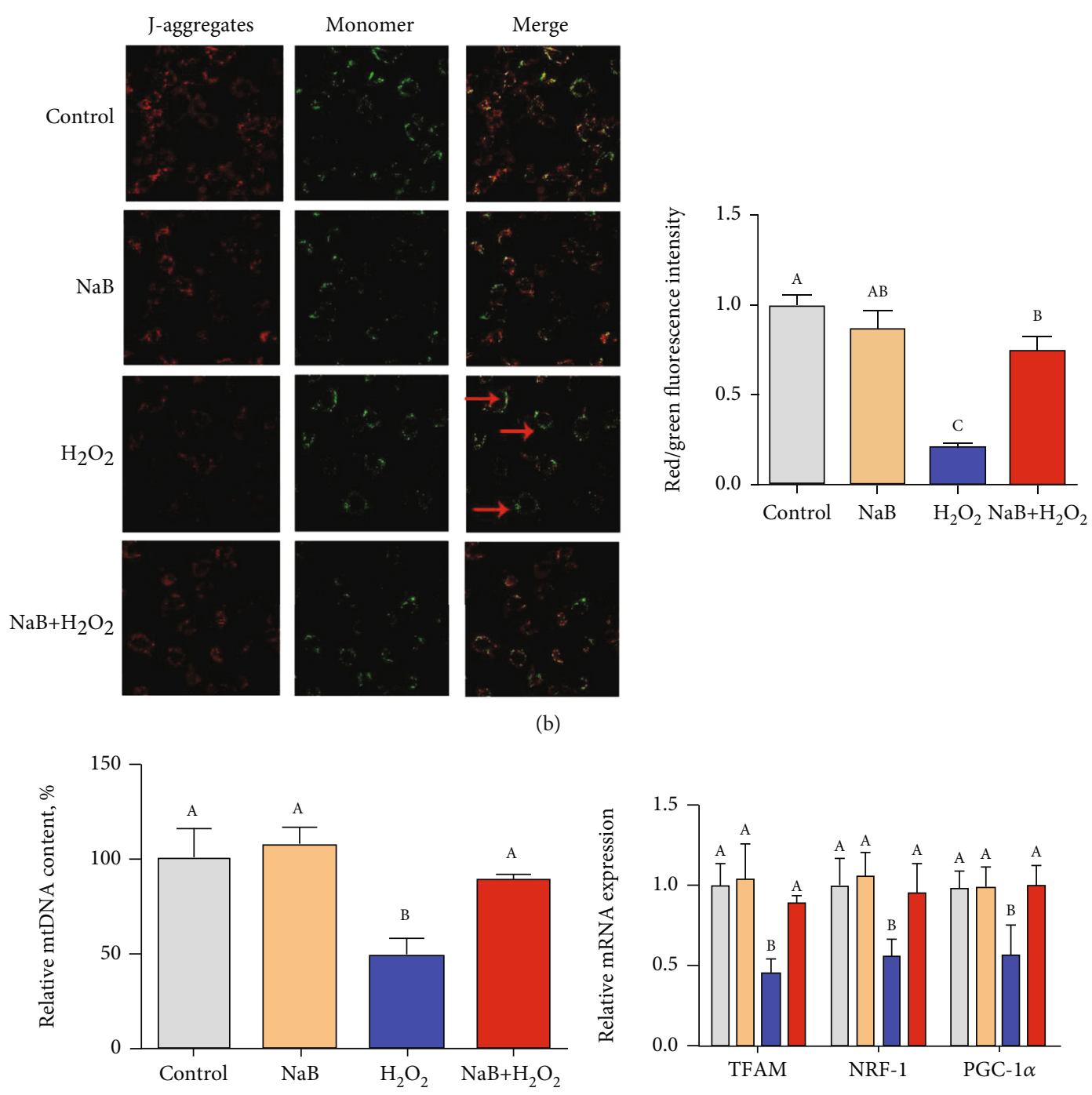

(b)

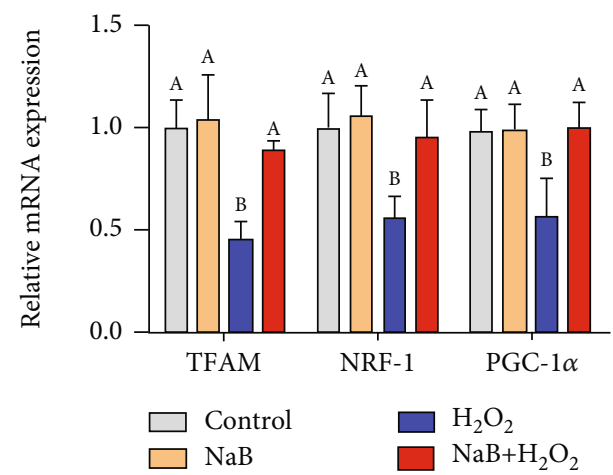

(c)

(d)

Figure 2: Continued. 

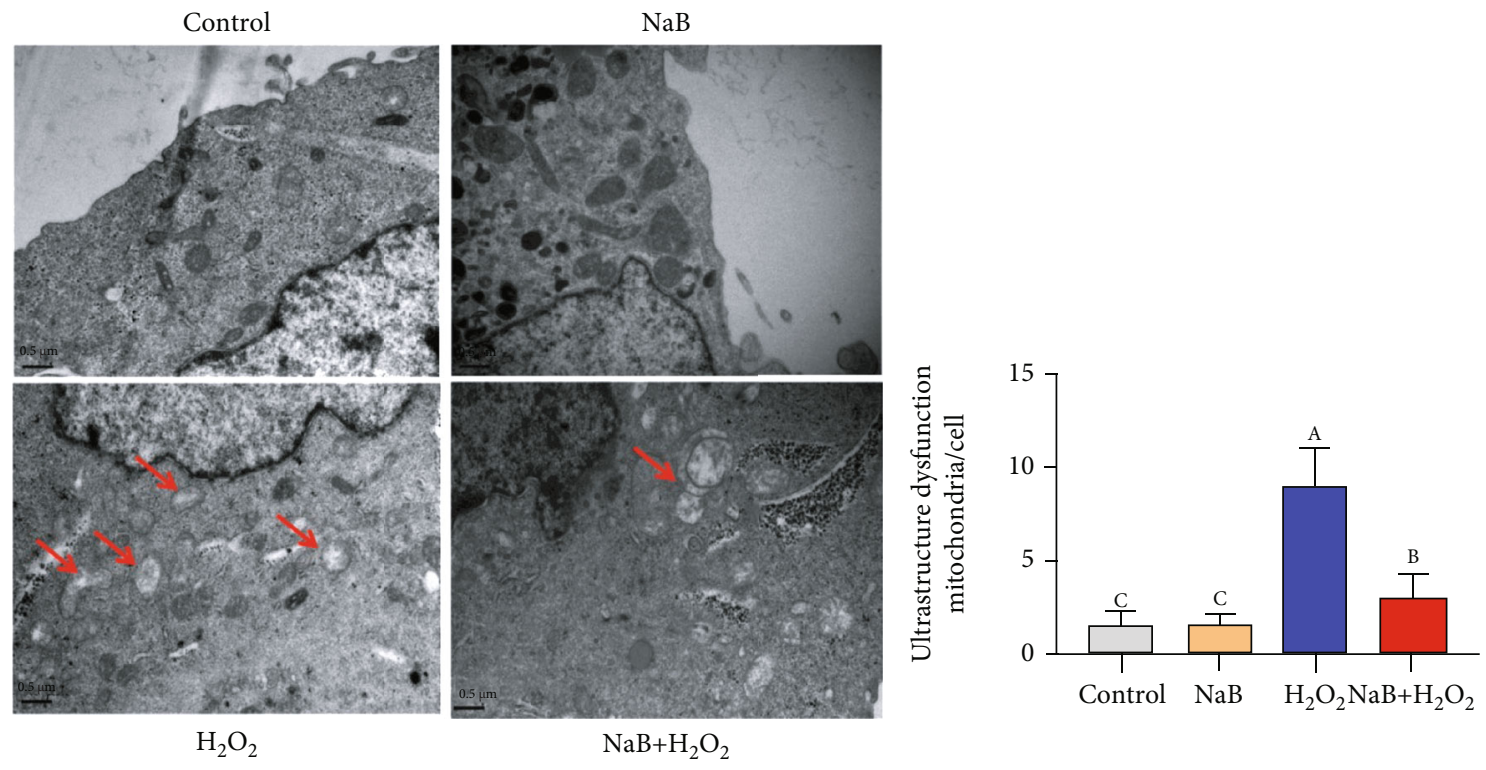

(e)
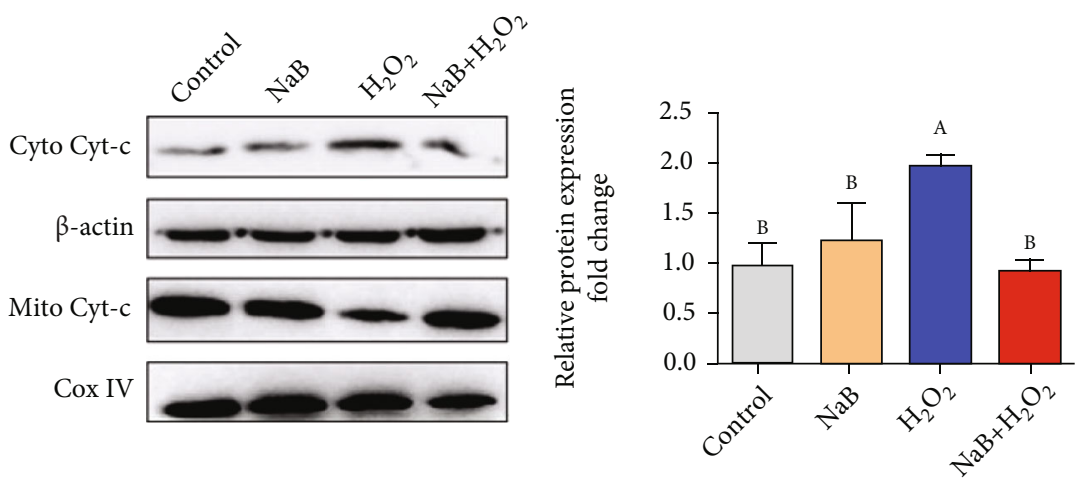

Cyto Cyt-c/ß-actin

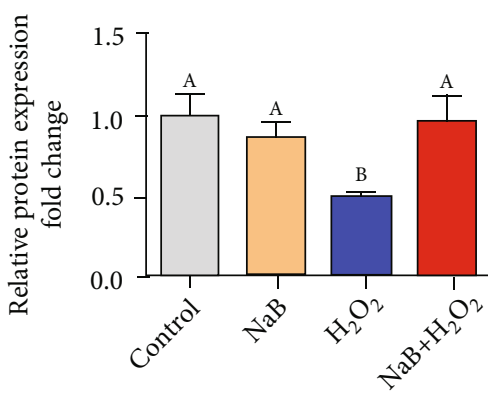

Mito cyt-c/Cox IV

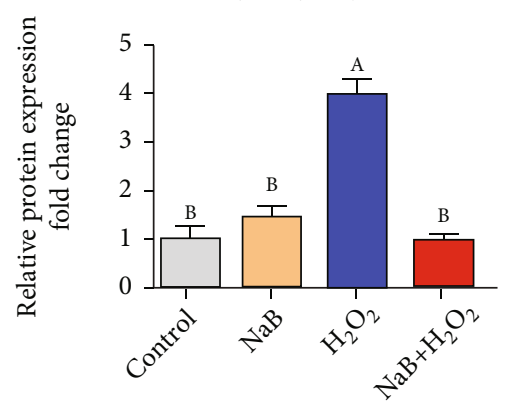

(Cyto Cyt-c/ß-actin) / (Mito Cyt-c/Cox IV)

(f)

FIGURE 2: Effects of sodium butyrate $(\mathrm{NaB})$ on the mitochondrial function of porcine intestinal epithelial cells (IPEC-J2) treated with H2O2. (a) Flow cytometry to detect changes in cell mitochondrial membrane potential. (b) The ratio of red to green of mitochondria JC-1 under a laser confocal microscope and quantification results. (c, d) Mitochondrial DNA (mtDNA) content and mitochondrial transcription factor A (TFAM), nuclear respiratory factor-1 (NRF-1), and peroxisomal proliferator-activated receptor-g coactivator-1 $\alpha$ (PGC-1 $\alpha)$ gene expression. (e) Ultrastructure of mitochondria under a transmission electron microscope (TEM). (f) Protein expression and quantification of cytochrome c. Different superscript letters within the same row mean significant difference $(P<0.05)$. 


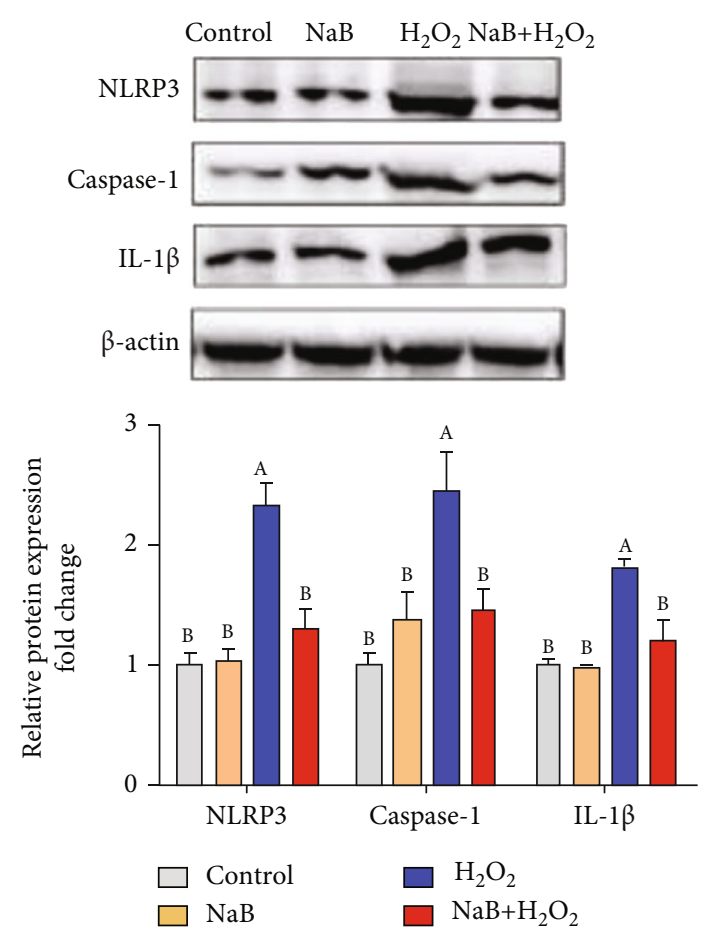

(a)

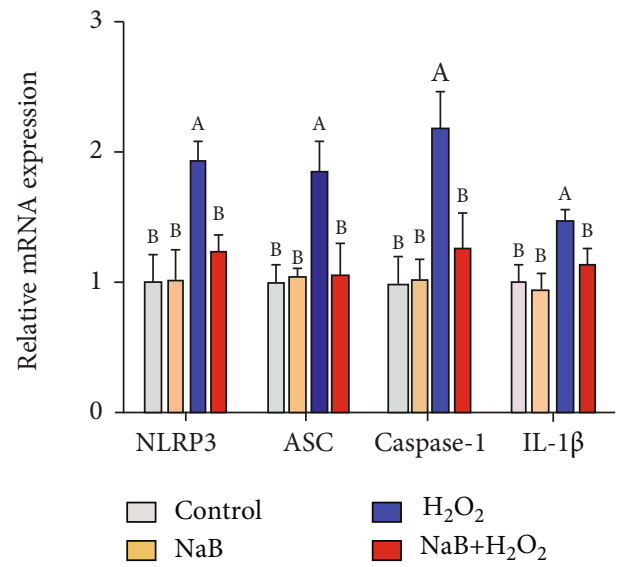

(b)

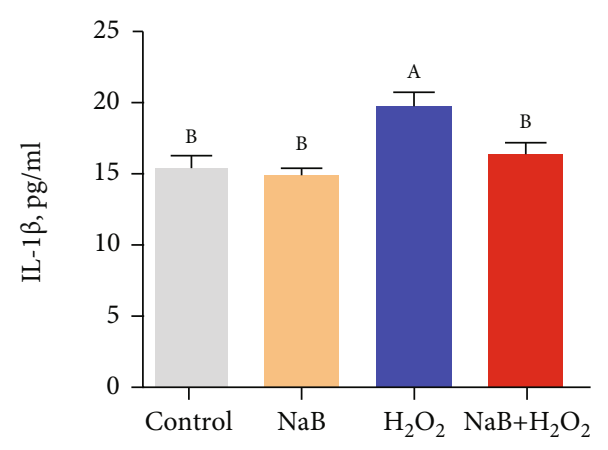

(c)

Figure 3: Effects of sodium butyrate $(\mathrm{NaB})$ on $\mathrm{H} 2 \mathrm{O} 2$ treatment of porcine intestinal epithelial cell (IPEC-J2) inflammasome and inflammatory factors. (a) Protein expression and quantification of recombinant NLR family, pyrin domain-containing protein 3 (NLRP3), Caspase-1, and IL-1 $\beta$. (b) Relative gene expression of NLRP3, Caspase-1, ASC, and IL-1 $\beta$. (c) The content of IL-1 $\beta$ in IPEC-J2 cells. Different superscript letters within the same row mean significant difference $(P<0.05)$.

distributed near the cell membrane and forms a continuous ring around the cell membrane; but compared with the control group, the $\mathrm{H}_{2} \mathrm{O}_{2}$ treatment destroyed the normal distribution and expression of Claudin-1 $(P<0.05)$. The sodium butyrate pretreatment prevented the disorder of Claudin-1 distribution at the cell boundary and the decrease of protein expression caused by $\mathrm{H}_{2} \mathrm{O}_{2}(P<0.05)$.

3.5. Effect of Sodium Butyrate on Mitophagy of IPEC-J2 Cells Treated with $\mathrm{H}_{2} \mathrm{O}_{2}$. As shown in Figures 5(a)-5(d), compared with the control group, $\mathrm{H}_{2} \mathrm{O}_{2}$ significantly increased the mRNA expression of LC3B, Beclin-1, ATG 5, PINK1, and Parkin of IPEC-J2 cells and reduced the mRNA level of P62 $(P<0.05)$. Compared with the $\mathrm{H}_{2} \mathrm{O}_{2}$ group, sodium butyrate pretreatment further increased its mRNA level $(P<0.05)$ and reduced the mRNA expression of $\mathrm{P} 62$
$(P<0.05)$. Similarly, compared with the $\mathrm{H}_{2} \mathrm{O}_{2}$ group, sodium butyrate pretreatment further increased the protein expression of PINK1, Parkin, LC3B, and Beclin-1 $(P<0.05$ ) and decreased the expression of P62 $(P<0.05)$. According to the immunofluorescence results (Figure 5(e)), we found that compared with the control group, $\mathrm{H}_{2} \mathrm{O}_{2}$ significantly increased the fluorescence intensity of LC3B of IPEC-J2 cells $(P<0.05)$; compared with the $\mathrm{H}_{2} \mathrm{O}_{2}$ group, sodium butyrate pretreatment further increased LC3B fluorescence intensity $(P<0.05)$. As shown in Figure 5(f), the results of confocal microscopy showed that under oxidative stress, sodium butyrate promotes the colocalization of LC3B and mitochondria and also increases the colocalization of PINK1, Parkin, and LC3B (Figures 5(g) and 5(h)). Similarly, according to the transmission electron microscope (TEM) results, we also observed the increase of mitophagy vesicles under 


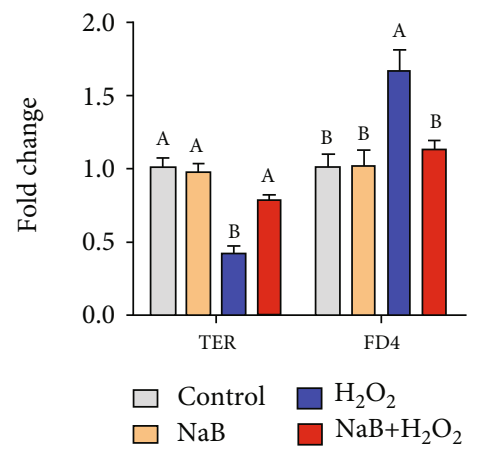

(a)
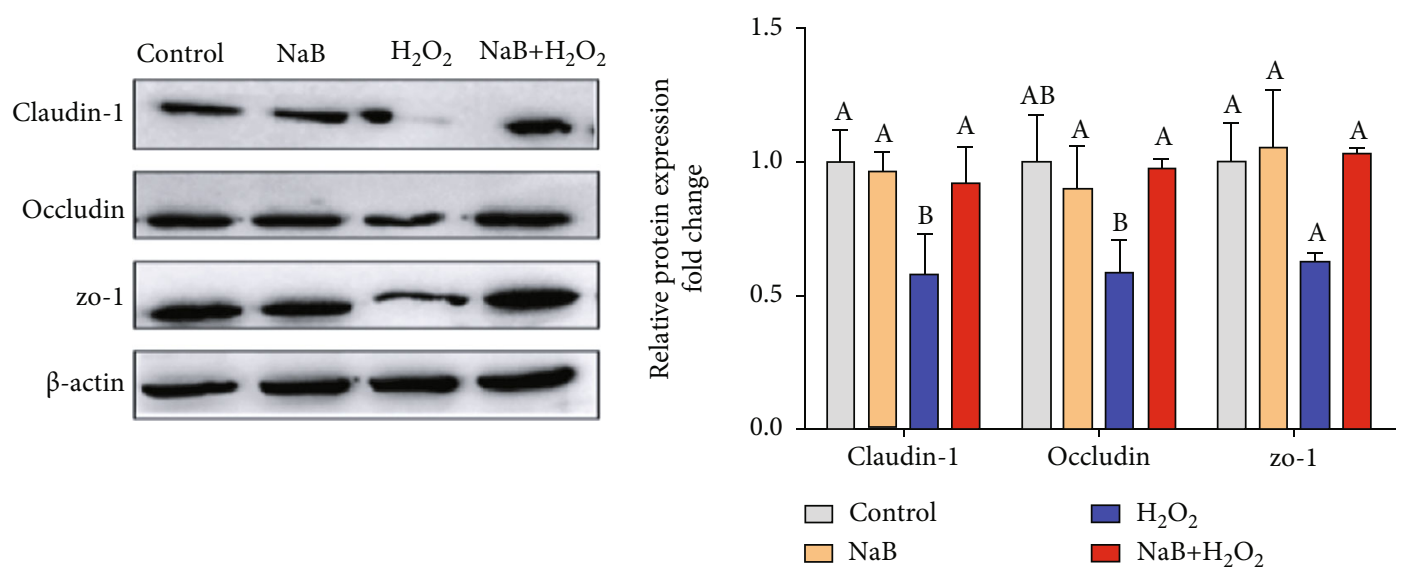

(b)
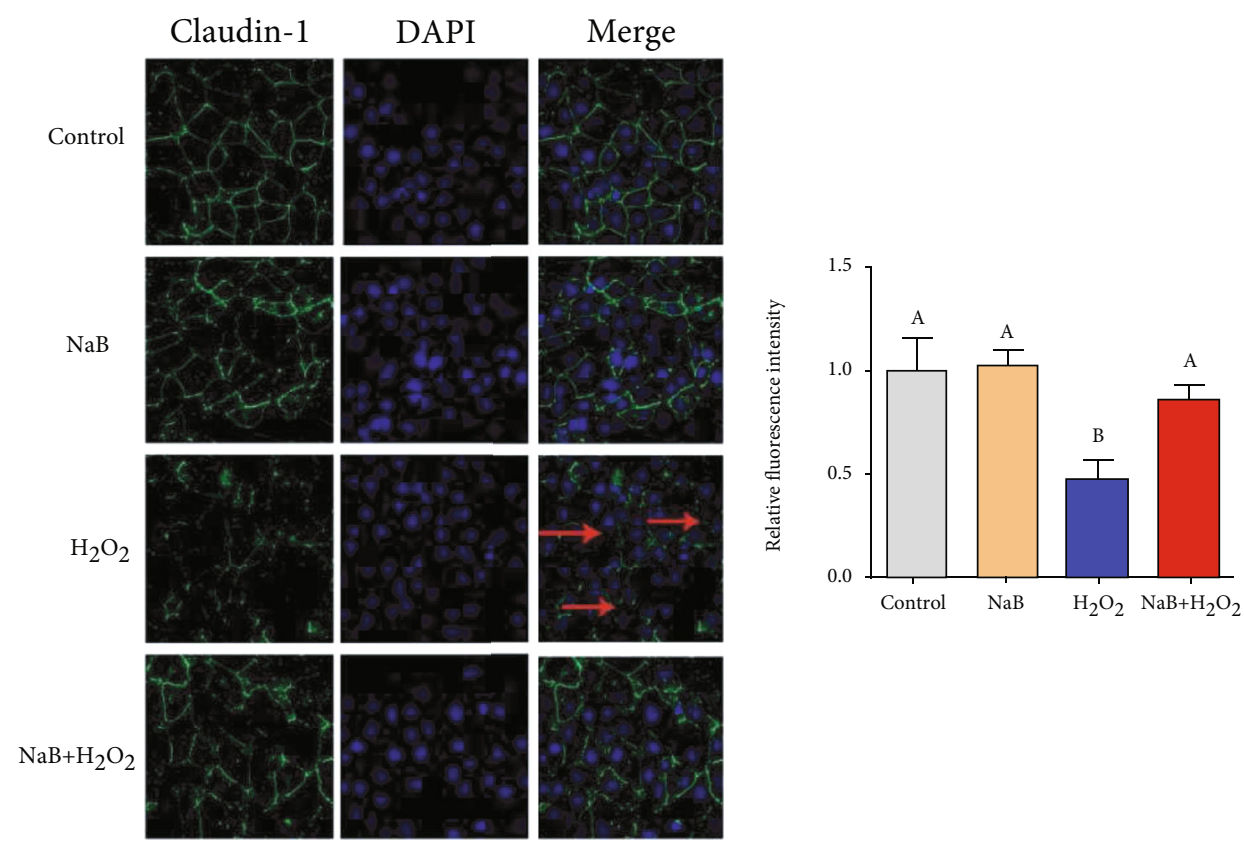

(c)

FIGURE 4: Effects of sodium butyrate $(\mathrm{NaB})$ on the barrier function of porcine intestinal epithelial cells (IPEC-J2) treated with hydrogen peroxide (H2O2). (a) Intestinal epithelial transepithelial resistance (TER) and fluorescein isothiocyanate dextran $4 \mathrm{kDa}$ (FD4) permeability. (b) Protein expression and quantification of Claudin-1, Occludin, and ZO-1. (c) The distribution and quantification of Claudin-1 protein under a confocal microscope. Different superscript letters within the same row mean significant difference $(P<0.05)$. 


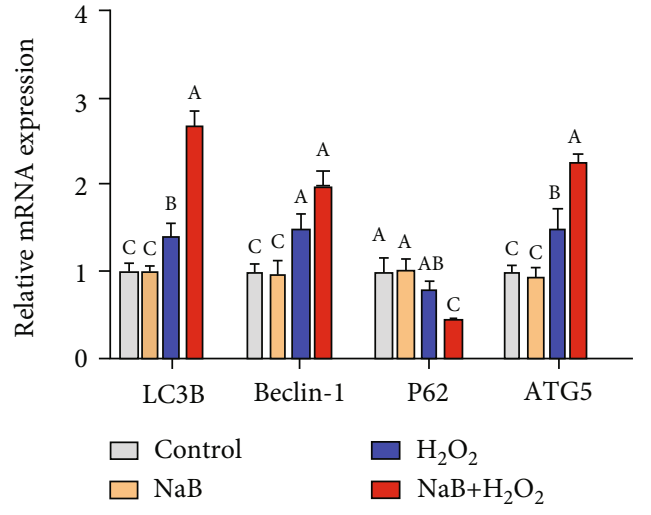

(a)
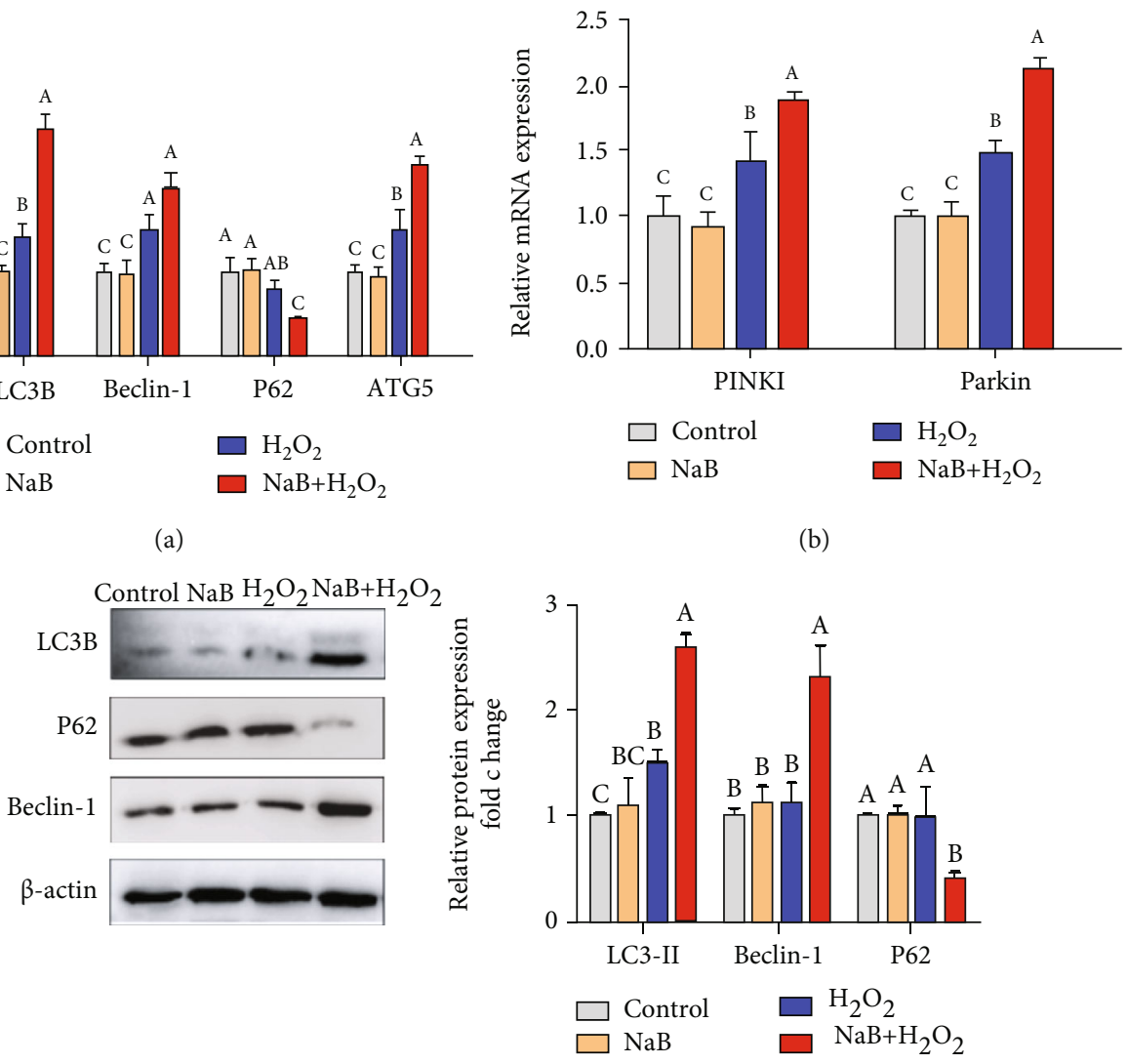

(b)

(c)
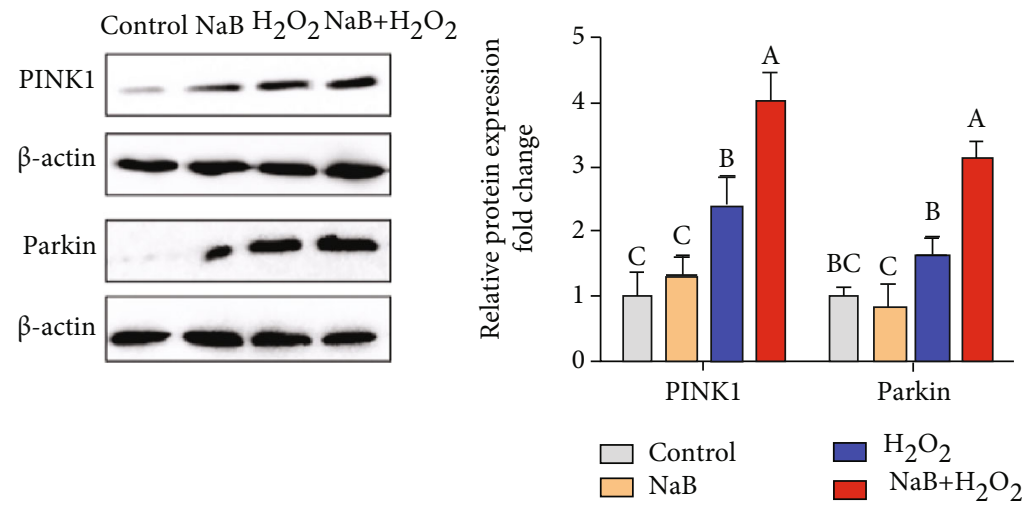

(d)

Figure 5: Continued. 

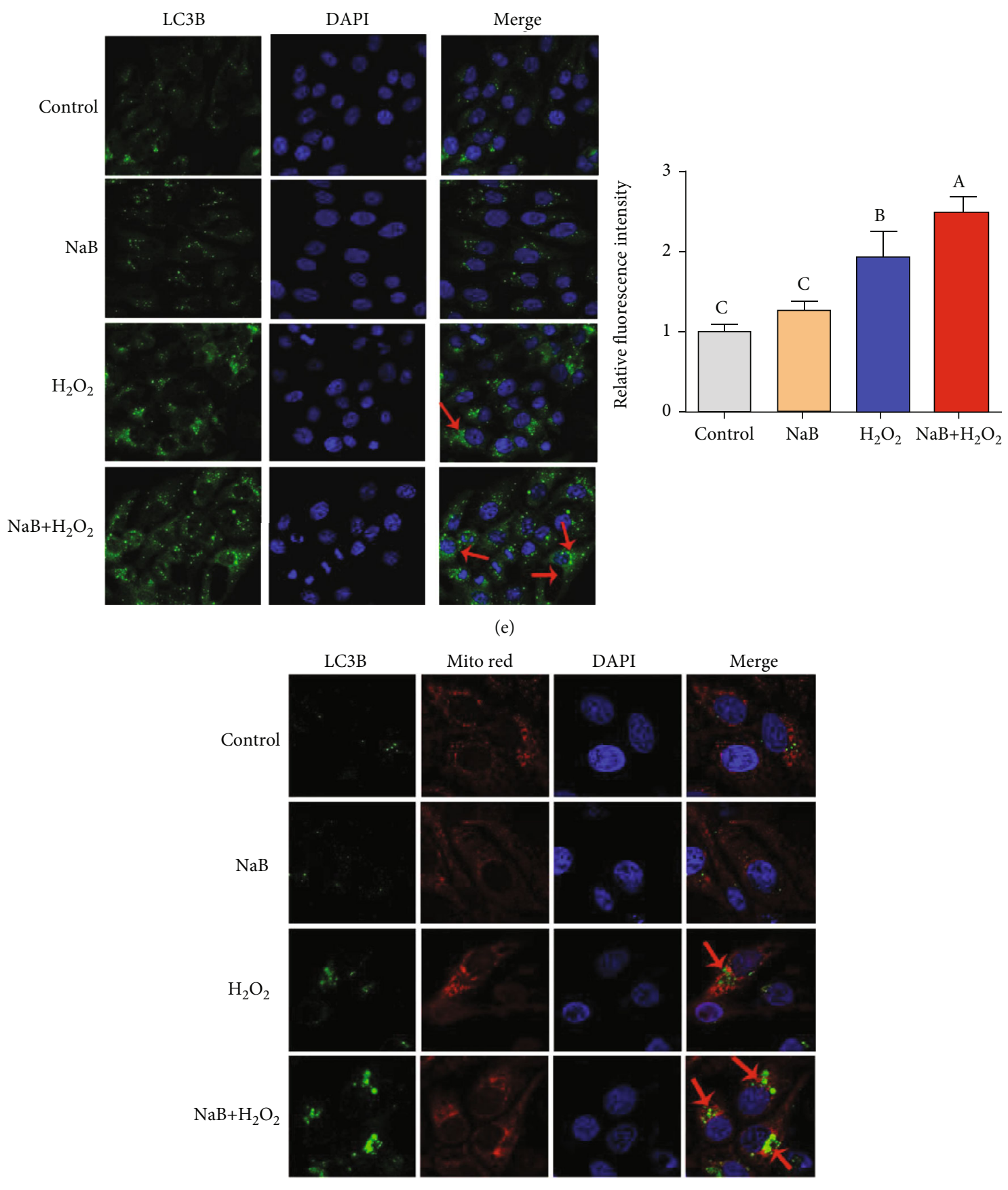

(f)

Figure 5: Continued. 


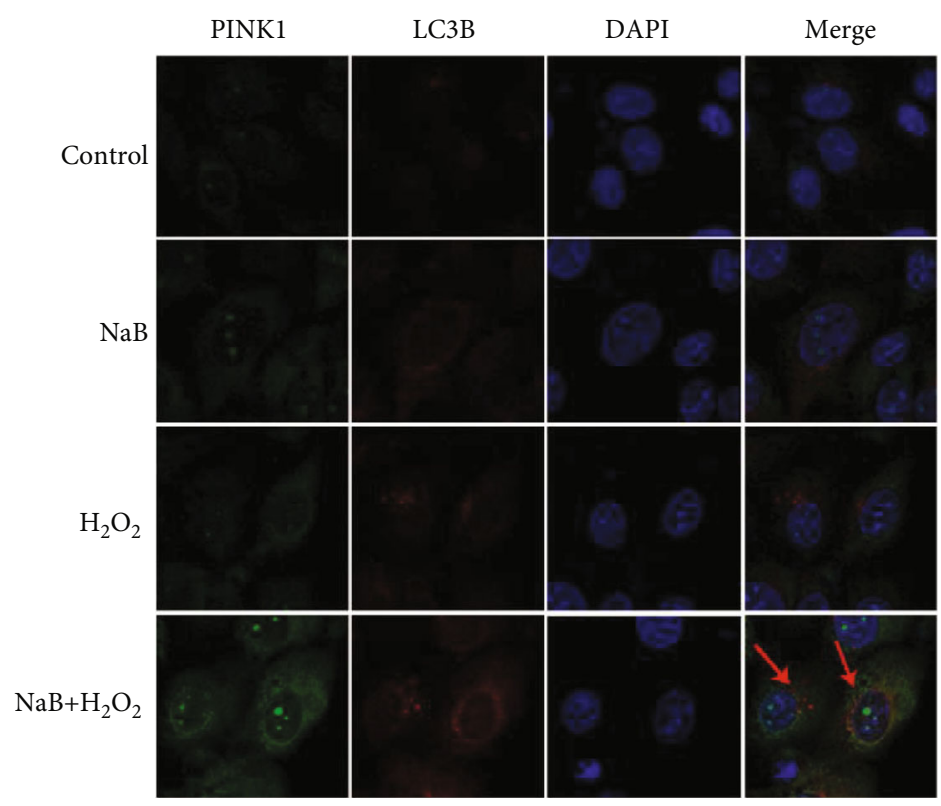

(g)

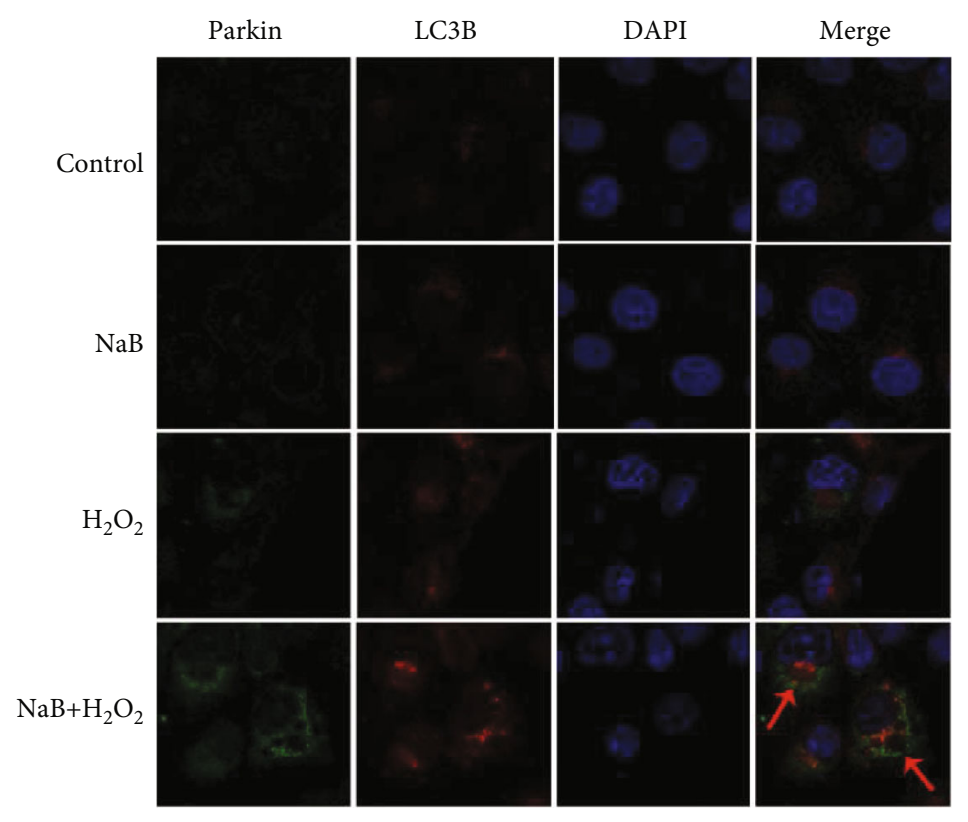

(h)

Figure 5: Continued. 


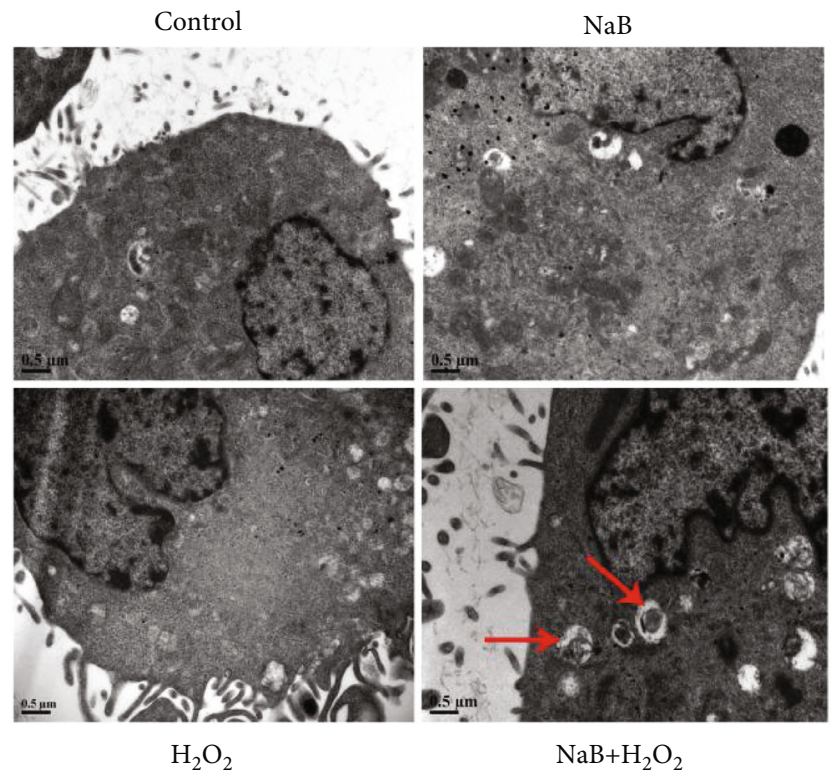

(i)

Figure 5: Effects of sodium butyrate (NaB) on mitophagy of porcine intestinal epithelial cells (IPEC-J2) treated with H2O2. (a, b) mRNA expression level of autophagy-related genes. (c, d) Western blot of autophagy-related protein expression and quantification. (e) The protein expression and quantification of light-chain-associated protein B (LC3B) under a laser confocal microscope. (f) Colocalization of mitochondrial Mito Red and autophagy protein LC3B under a laser confocal microscope. (g) Colocalization of mitophagy proteins PINK1 and LC3B under a laser confocal microscope. (h) Colocalization of mitophagy proteins Parkin and LC3B under a laser confocal microscope. (i) Mitophagy vesicles under transmission electron microscope. Different superscript letters within the same row mean significant difference $(P<0.05)$.

the cotreatment of sodium butyrate and $\mathrm{H}_{2} \mathrm{O}_{2}$ (Figure 5(i)). These results indicate that sodium butyrate promotes mitophagy in $\mathrm{H}_{2} \mathrm{O}_{2}$-treated IPEC-J2 cells.

3.6. The Effect of Sodium Butyrate on Oxidative Stress, Intestinal Epithelium Barrier, and Mitophagy of IPEC-J2 Cells after Inhibition of Mitophagy or AMPK $\alpha$. As shown in Figures 6(a)-6(c), in comparison with the $\mathrm{H}_{2} \mathrm{O}_{2}$ group, sodium butyrate increased the SOD activity $(P<0.05)$ and decreased the MDA level $(P<0.05)$. Compared with the $\mathrm{NaB}+\mathrm{H}_{2} \mathrm{O}_{2}$ group, Mdivi-1 or Compound $\mathrm{C}$ treatment increased the MDA level $(P<0.05)$ and inhibited the SOD activity $(P<0.05)$. These results indicate that inhibition of mitophagy and AMPK $\alpha$ weakens the antioxidant effect of sodium butyrate. As shown in Figure 6(d), compared with the $\mathrm{NaB}+\mathrm{H}_{2} \mathrm{O}_{2}$ group, Mdivi-1 and Compound $\mathrm{C}$ treatments significantly increased $(P<0.05)$ the percentage of depolarized cells (reflecting the decrease in mitochondrial membrane potential). Compared with the $\mathrm{NaB}+\mathrm{H}_{2} \mathrm{O}_{2}$ group, Mdivi-1 and Compound $\mathrm{C}$ treatments significantly increased the ROS levels of IPEC-J2 cells $(P<0.05)$ (Figure 6(e)). These results indicate that inhibition of mitophagy or AMPK $\alpha$ weakens the protective effects of sodium butyrate on mitochondria under oxidative stress. As shown in Figures 6(f) and 6(g), compared with the $\mathrm{NaB}$ $+\mathrm{H}_{2} \mathrm{O}_{2}$ group, Mdivi-1 or Compound $\mathrm{C}$ treatment significantly reduced the TER $(P<0.05)$ and increased FD4 $(P<0.05)$. Mdivi-1 or Compound $\mathrm{C}$ treatment also reduced the expression of tight junction proteins Claudin-1, Occlu- din, and ZO-1 $(P<0.05)$. The data indicated that inhibition of mitophagy or AMPK $\alpha$ weakened the protective effects of sodium butyrate on intestinal epithelium barrier function under oxidative stress.

3.7. The Effect of Sodium Butyrate on the Mitophagy of IPECJ2 Cells after Inhibiting Mitophagy or AMPK $\alpha$. As shown in Figures 7(a) and 7(b), compared with the $\mathrm{NaB}+\mathrm{H}_{2} \mathrm{O}_{2}$ group, Mdivi-1 or Compound $\mathrm{C}$ treatment significantly reduced Beclin-1 levels $(P<0.05)$ and increased P62 levels $(P<0.05$ ). Similarly, compared with the $\mathrm{NaB}+\mathrm{H}_{2} \mathrm{O}_{2}$ group, Mdivi-1 or Compound $\mathrm{C}$ treatment also reduced the expression levels of mitophagy proteins PINK1 and Parkin $(P<0.05)$. Immunofluorescence result showed that inhibition of mitophagy or AMPK $\alpha$ reduced the colocalization of LC3B and mitochondria and reduced the colocalization of PINK1 and LC3B (Figures 7(c) and 7(d)). These results indicate that inhibition of mitophagy and AMPK $\alpha$ suppresses the induction of mitophagy by sodium butyrate.

3.8. The Effect of Sodium Butyrate on Mitophagy, Oxidative Stress, and Intestinal Epithelium Barrier of IPEC-J2 Cells after Interference with $A M P K \alpha$. As shown in Figure 8(a), interference with $\mathrm{AMPK} \alpha$ reduced $(P<0.05)$ the protein expression of PINK1 and Parkin and increased the protein expression level of P62 $(P<0.05)$ when compared with the $\mathrm{H}_{2} \mathrm{O}_{2}$ group and the $\mathrm{NaB}+\mathrm{H}_{2} \mathrm{O}_{2}$ group. The results indicated that interference with $\mathrm{AMPK} \alpha$ blocked sodium butyrateinduced enhancement of mitophagy. As shown in 


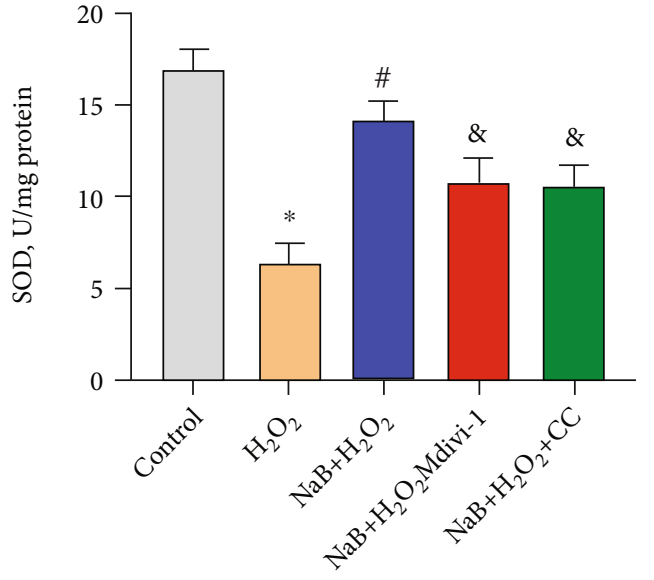

(a)

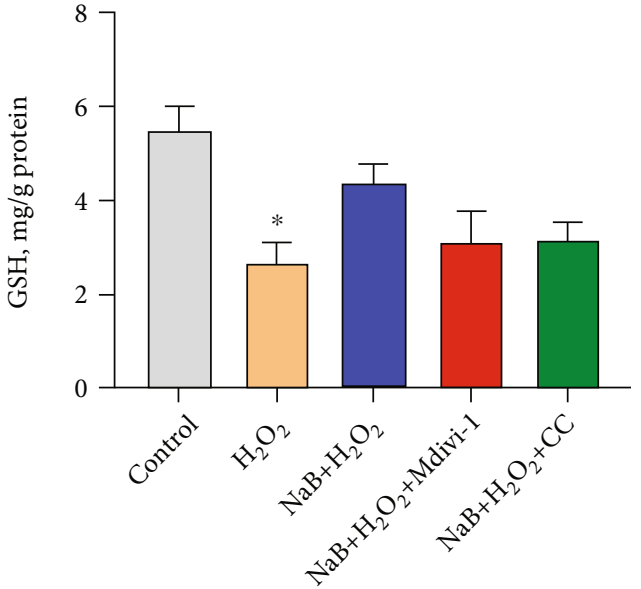

(b)

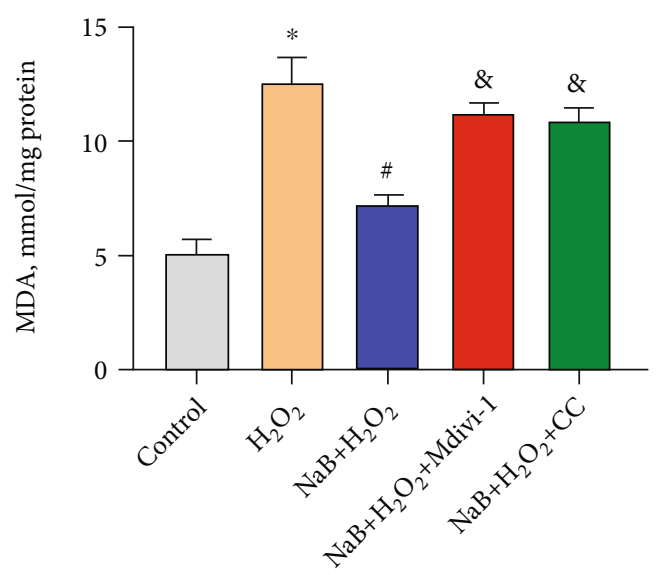

(c)

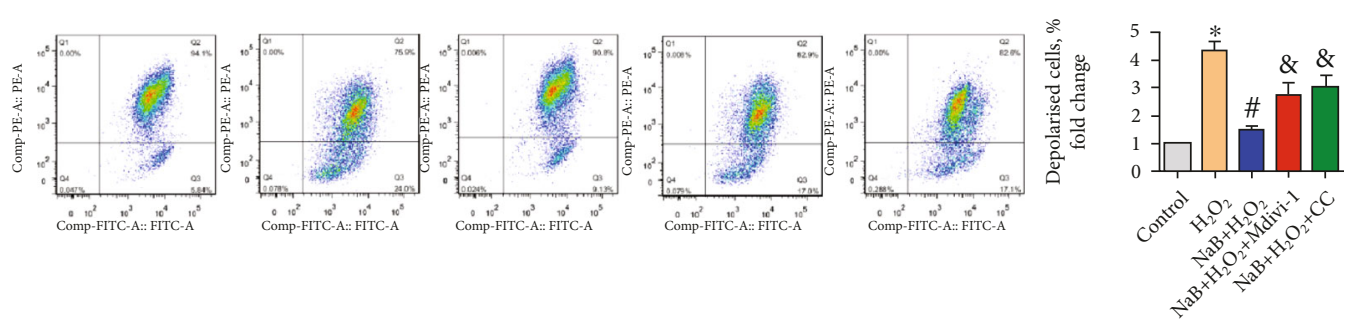

(d)

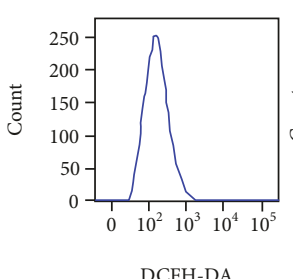

DCFH-DA

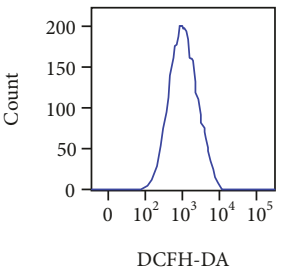

DCFH-DA

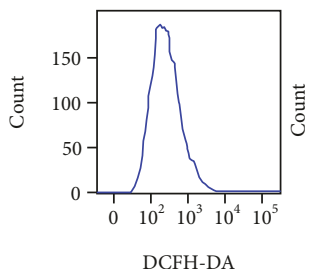

DCFH-DA

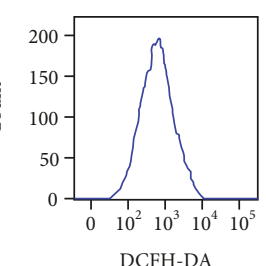

(e)

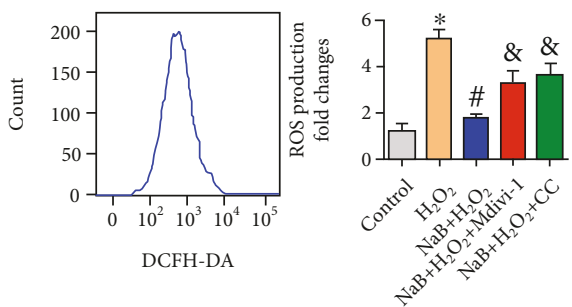

DCFH-DA

Figure 6: Continued. 


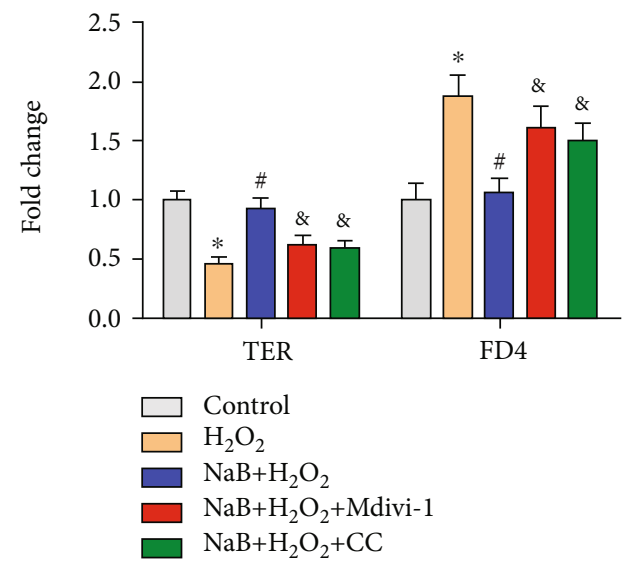

(f)
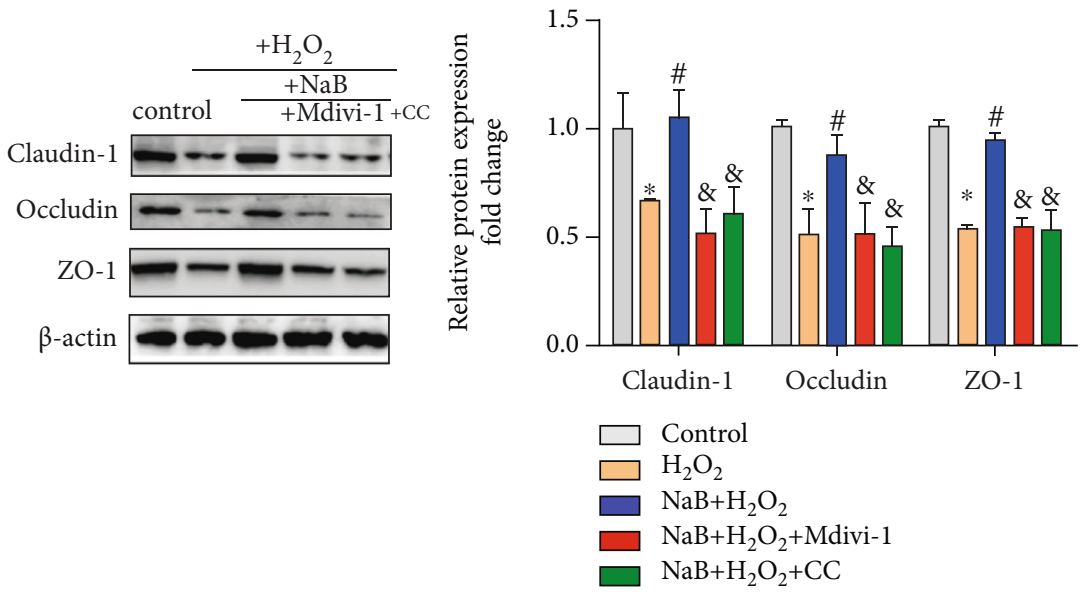

(g)

Figure 6: Effects of sodium butyrate $(\mathrm{NaB})$ on oxidative stress, intestinal epithelium barrier, and mitophagy of porcine intestinal epithelial cells (IPEC-J2) after inhibiting mitophagy or AMPK. (a-c) Superoxide dismutase (SOD), glutathione reductase (GSH) activity, and malondialdehyde (MDA) content of IPEC-J2 treated with Mdivi-1 or Compound C (CC). (d) Cellular mitochondrial membrane potential quantification by flow cytometry. (e) Cellular reactive oxygen species (ROS) level quantification by flow cytometry. (f) Intestinal epithelial transepithelial resistance (TER) and fluorescein isothiocyanate dextran $4 \mathrm{kDa}$ (FD4) permeability. (g) Protein expression and quantification of tight junction Claudin-1, Occludin, and ZO-1. * indicates a significant difference compared with the control group $(P<0.05)$; \# indicates a significant difference compared with the $\mathrm{H} 2 \mathrm{O} 2$ group $(P<0.05)$; \& indicates a significant difference compared with the $\mathrm{NaB}+\mathrm{H} 2 \mathrm{O} 2$ group $(P<0.05)$.

Figures $8(\mathrm{~b})-8(\mathrm{f})$, the results showed that interference with AMPK $\alpha$ significantly reduced the activity of SOD and increased the level of MDA $(P<0.05)$ when compared with the $\mathrm{NaB}+\mathrm{H}_{2} \mathrm{O}_{2}$ group. The interference with AMPK $\alpha$ increased the production of ROS $(P<0.05)$ and decreased the mitochondrial membrane potential $(P<0.05)$ when compared with the $\mathrm{H}_{2} \mathrm{O}_{2}$ group and the $\mathrm{NaB}+\mathrm{H}_{2} \mathrm{O}_{2}$ group, indicating that the role of sodium butyrate on alleviating cellular oxidative stress and mitochondrial dysfunction was counteracted. From the results, it could be concluded that AMPK $\alpha$-mediated mitophagy is necessary for sodium butyrate's protective effects on cellular redox status and mitochondrial function. As shown in Figures 8(g) and 8(h), interference with AMPK $\alpha$ significantly increased the protein expression of NLRP3 and Caspase-1 when compared with $\mathrm{H}_{2} \mathrm{O}_{2}$ and $\mathrm{NaB}+\mathrm{H}_{2} \mathrm{O}_{2}$ groups $(P<0.05)$, indicating that interference with AMPK $\alpha$ significantly reduced the inhibition of NLRP3 activation by sodium butyrate. At the same time, interference with $\mathrm{AMPK} \alpha$ resulted in a decrease in TER $(P>0.05)$ and an increase in FD4 $(P>0.05)$, indicating that interference with AMPK $\alpha$ weakened the protective effect of sodium butyrate on the intestinal epithelium barrier. From the results, it could be concluded that AMPK $\alpha$ is necessary for sodium butyrate to inhibit intestinal inflammation and protect intestinal epithelium barrier of IPEC-J2 cells under oxidative stress.

\section{Discussion}

$\mathrm{Ma}$ et al. reported that sodium butyrate could alleviate the intestinal epithelium barrier damage of IPEC-J2 cells and regulate cellular antioxidant level [8]. So far, no data was found on the effect of sodium butyrate on oxidative stress- induced intestine damage in IPEC-J2 cells. The present study demonstrated that sodium butyrate protected IPEC$\mathrm{J} 2$ cells from $\mathrm{H}_{2} \mathrm{O}_{2}$-induced oxidative stress, as indicated by the increased SOD and GSH activities and the decreased MDA and ROS level in IPEC-J2 cells. Similarly, Russo et al. found that butyrate could effectively inhibit the decrease of glutathione transferase (GST) activity and the increase of ROS level induced by LPS in Caco- 2 cells [27]. Excessive production of ROS would affect the electronic respiration chain of mitochondria, open mitochondrial permeability transition pores, and lead to depolarization of mitochondrial membranes [28]. We found that sodium butyrate alleviated $\mathrm{H}_{2} \mathrm{O}_{2}$-induced decrease in mitochondrial membrane potential (MMP), as indicated by the decreased proportion of depolarized cells and the increased ratio of red (JC-1 polymer)/green (JC-1 polymer) fluorescence intensity when compared with the $\mathrm{H}_{2} \mathrm{O}_{2}$ group. Consistent with our research, $\mathrm{Li}$ et al. reported that sodium butyrate inhibited LPS-induced MMP loss of bovine mammary epithelial cells [29]. Increased ROS production in mitochondria and decreased MMP in mitochondria can lead to mitochondrial DNA damage, which could be related to abnormal mitochondrial biogenesis [28]. We found that sodium butyrate alleviated mitochondrial dysfunction by increasing mtDNA and mRNA expression levels of mitochondrial functionrelated genes TFAM, NRF-1, and PGC- $1 \alpha$. Similarly, a previous study found that sodium butyrate alleviated the oxidative damage of HepG2 cells, as indicated by increasing mtDNA copy number and the mRNA expression of PGC$1 \alpha$ and TFAM [30]. Changes in mitochondrial function will be accompanied by changes in mitochondrial ultrastructure. Our research showed that $\mathrm{H}_{2} \mathrm{O}_{2}$ caused obvious mitochondrial swelling, respiratory ridge breakage, and mitochondrial 

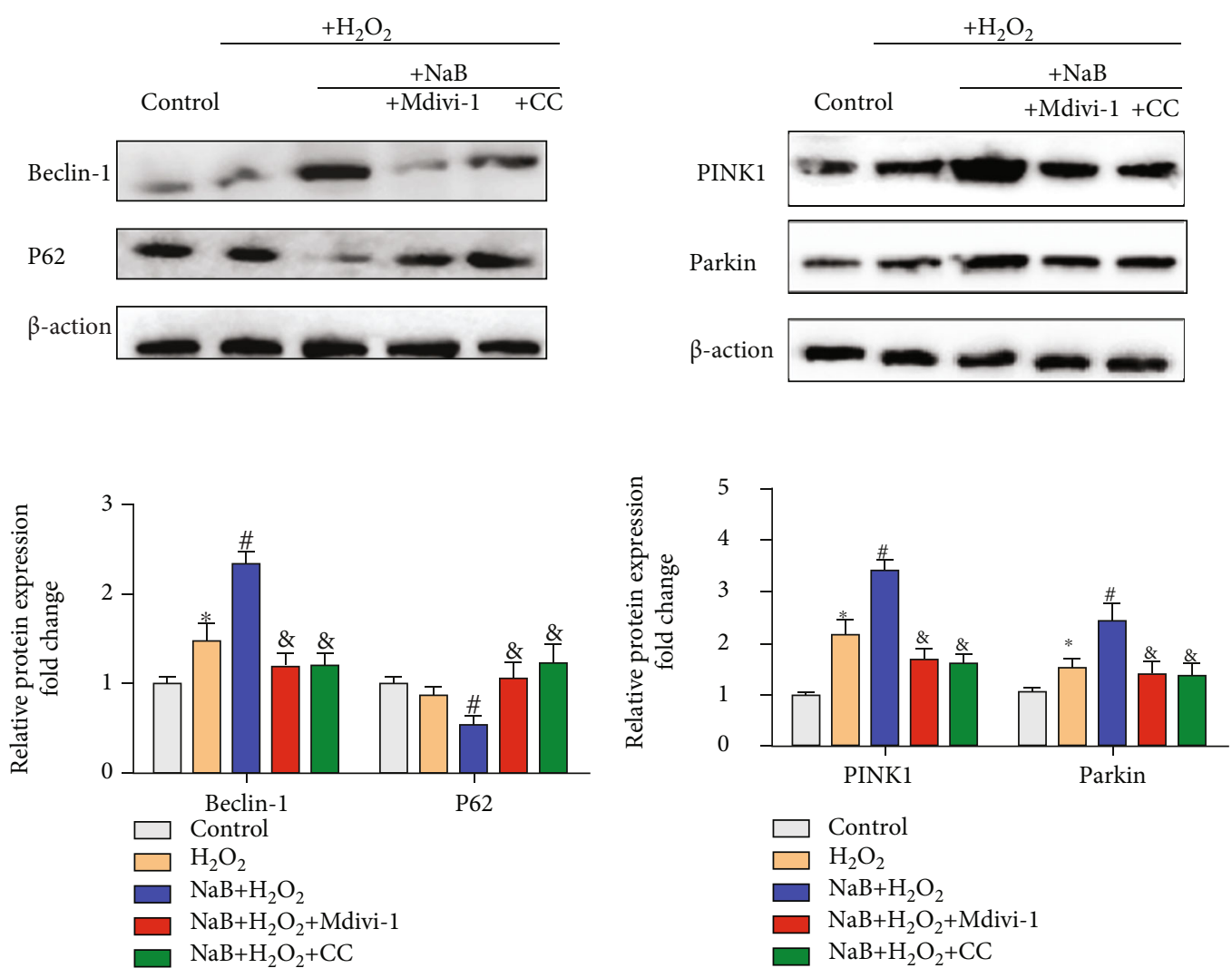

(a)

(b)

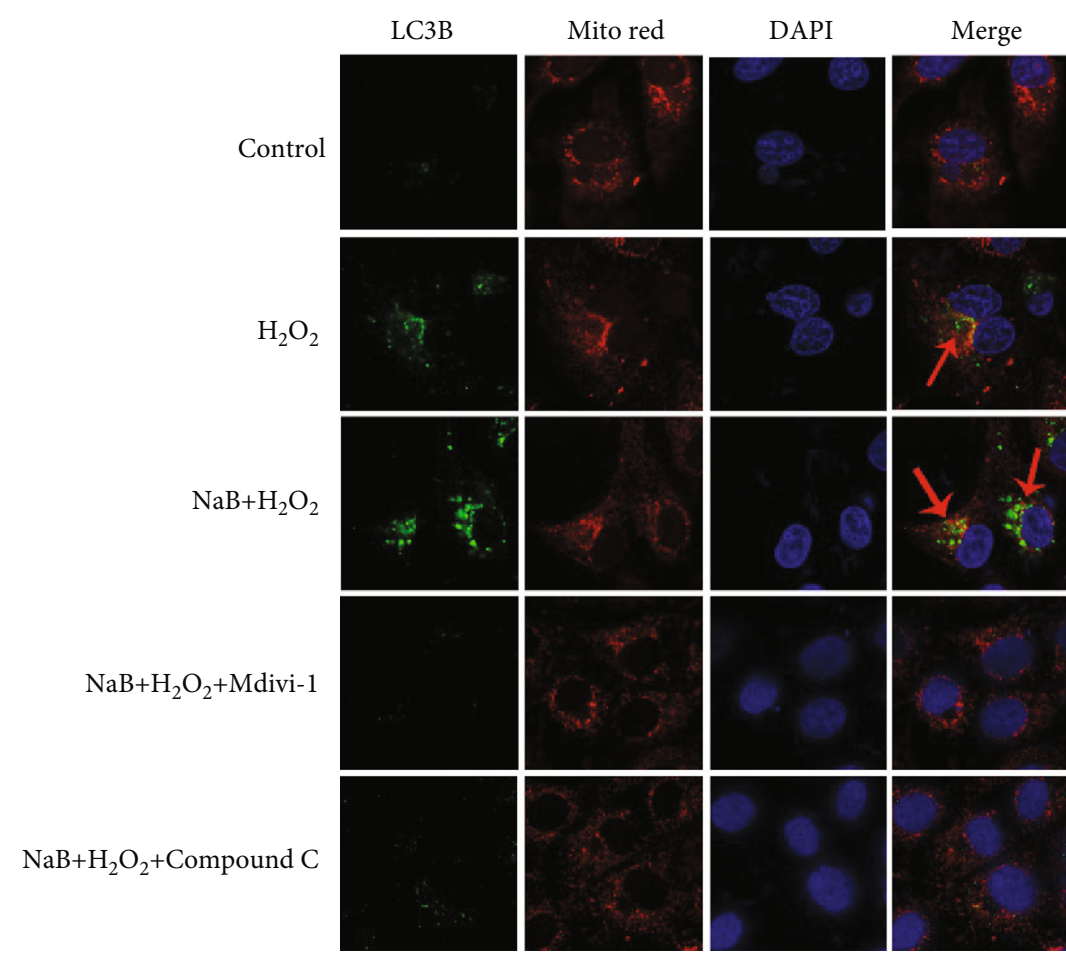

(c)

Figure 7: Continued. 


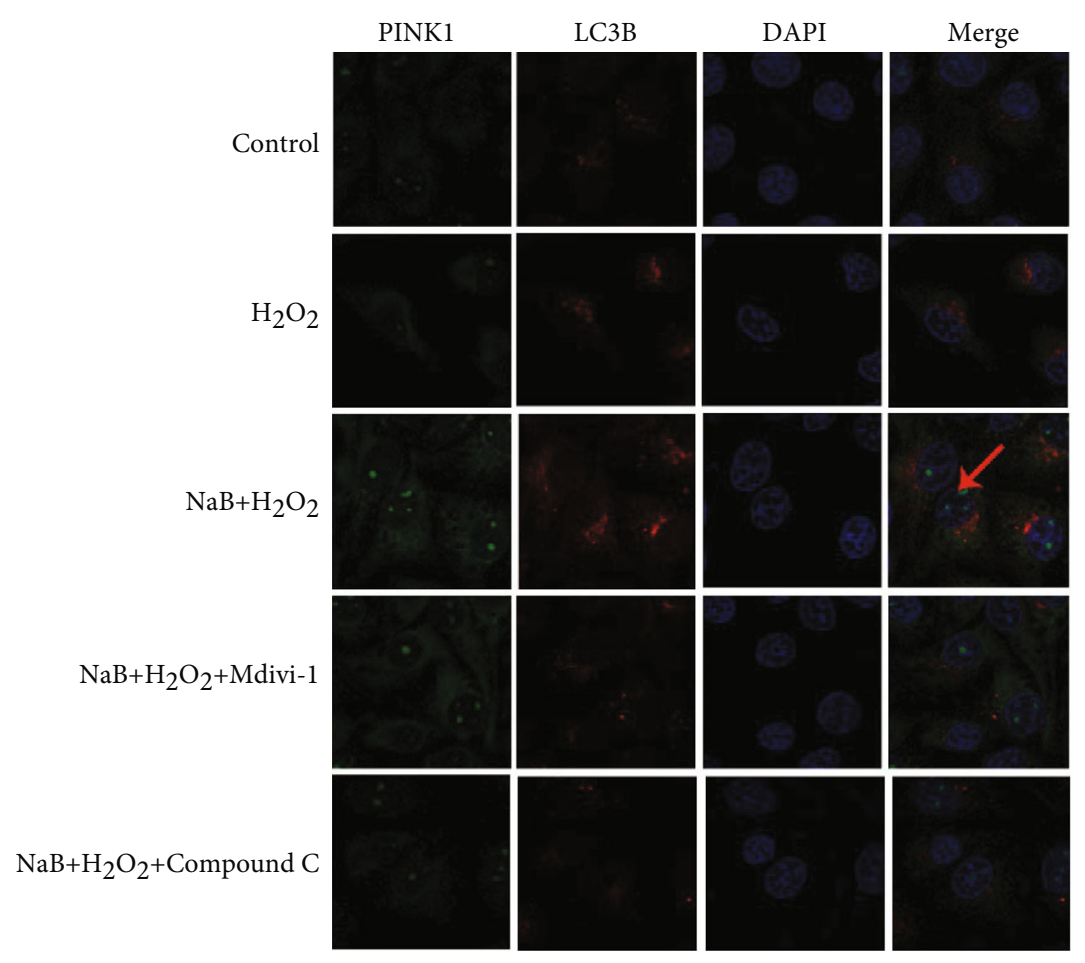

(d)

FIGURE 7: Effects of sodium butyrate $(\mathrm{NaB})$ on mitophagy of porcine intestinal epithelial cells (IEPC-J2) after inhibition of mitophagy or AMPK. ( $a, b)$ Protein expression and quantification of autophagy protein. (c) Colocalization of mitochondria and light-chain-associated protein B (LC3B) under a laser confocal microscope. (d) Colocalization of mitophagy proteins PINK1 and LC3B under a laser confocal microscope. * indicates a significant difference compared with the control group $(P<0.05)$; \# indicates a significant difference compared with the $\mathrm{H} 2 \mathrm{O} 2$ group $(P<0.05)$; \& indicates a significant difference compared with the $\mathrm{NaB}+\mathrm{H} 2 \mathrm{O} 2$ group $(P<0.05)$.

vacuolation while sodium butyrate pretreatment significantly improved the ultrastructure of mitochondria. The changes in the ultrastructure of mitochondria are not only the structural basis of mitochondrial oxidative damage but also lead to the release of Cyt $\mathrm{c}$ from mitochondria into the cytoplasm. We found that $\mathrm{H}_{2} \mathrm{O}_{2}$ increased Cyt $c$ level in the cytoplasm and decreased Cyt c level in mitochondria, suggesting that $\mathrm{H}_{2} \mathrm{O}_{2}$ increased the permeability of the inner and outer mitochondrial membranes, causing the release of mitochondrial Cyt $\mathrm{c}$ into the cytoplasm. Meanwhile, sodium butyrate alleviated the release of mitochondrial Cyt $\mathrm{c}$ induced by $\mathrm{H}_{2} \mathrm{O}_{2}$, indicating that sodium butyrate treatment could help improve mitochondrial function under oxidative stress. The above results revealed that sodium butyrate could effectively alleviate $\mathrm{H}_{2} \mathrm{O}_{2}$-induced redox status imbalance and mitochondrial impairment.

The intestinal barrier is an important line of defense for the body to prevent harmful substances from invading the body, and maintaining its integrity is vital to the health of the body [31, 32]. We found that sodium butyrate significantly alleviated $\mathrm{H}_{2} \mathrm{O}_{2}$-intestinal epithelium barrier injury of IPEC-J2 cells, as indicated by increasing intestinal epithelium TER and decreasing FD4 permeability. Similarly, Valenzano et al. and Wang et al. found that treatment of Caco-2 cells with a certain concentration of butyric acid could effectively improve the intestinal epithelium barrier $[33,34]$. Sodium butyrate also significantly inhibited the decrease of Claudin-1, Occludin, and ZO-1 in IPEC-J2 cells induced by $\mathrm{H}_{2} \mathrm{O}_{2}$. Consistent with our research, Yan and Ajuwon found that sodium butyrate increased the protein level of Claudin-3 and Claudin-4, thereby alleviating the damage of LPS to the integrity of IPEC-J2 monolayer cells [35]. The integrity of intestinal epithelial cells is not only related to the expression level of tight junction protein but also affected by its distribution area. In our study, we found that after $\mathrm{H}_{2} \mathrm{O}_{2}$ treatment, the normal distribution and expression of Claudin-1 were destroyed and sodium butyrate prevented the disorder of Claudin-1 distribution and the decrease of Claudin- 1 expression caused by $\mathrm{H}_{2} \mathrm{O}_{2}$. Similarly, studies found that when Caco- 2 cells were adversely stimulated, the Occludin and ZO-1 proteins originally expressed on the cell membrane would be transferred to the cytoplasm, thereby increasing cell permeability; however, butyric acid promoted tight junction protein redistribute and increased the expression level of tight junction $[9,36]$. These results indicated that sodium butyrate could effectively alleviate the intestinal epithelium barrier damage and the disorder of tight junction protein expression and distribution induced by $\mathrm{H}_{2} \mathrm{O}_{2}$ in IPEC-J2 cells.

Oxidative stress could cause intestinal inflammation, as proved by that excessive ROS production in the intestines of patients with ulcerative colitis and Crohn disease weakened antioxidant capacity and aggravated oxidative damage [27]. According to our results, $\mathrm{H}_{2} \mathrm{O}_{2}$ increased the mRNA 


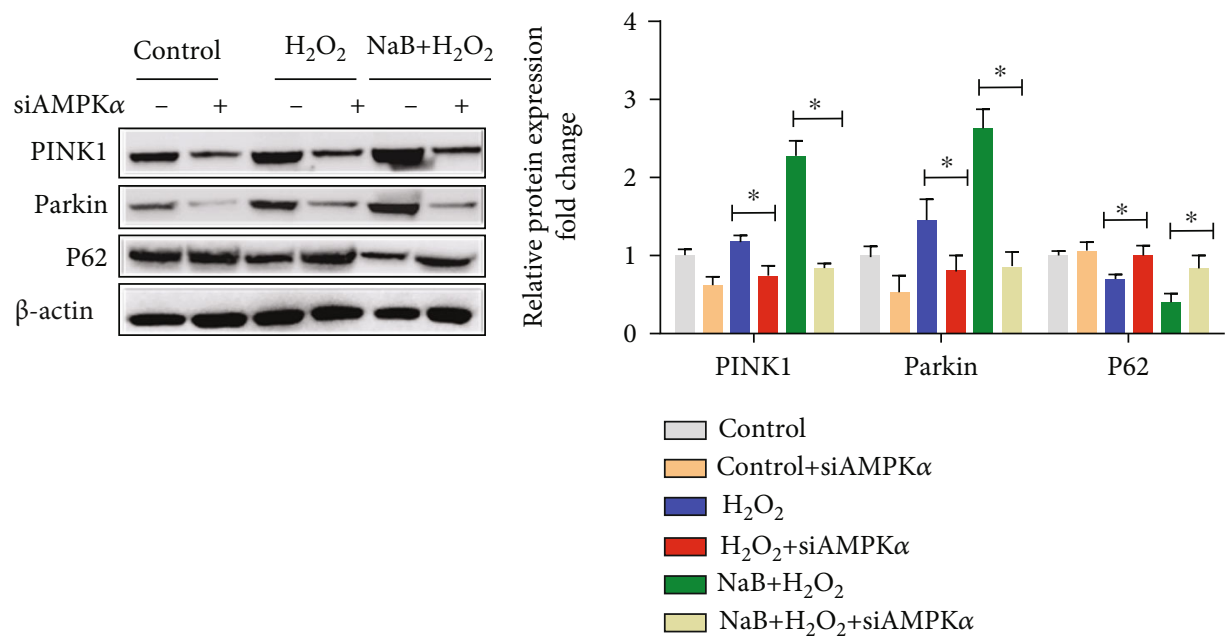

(a)

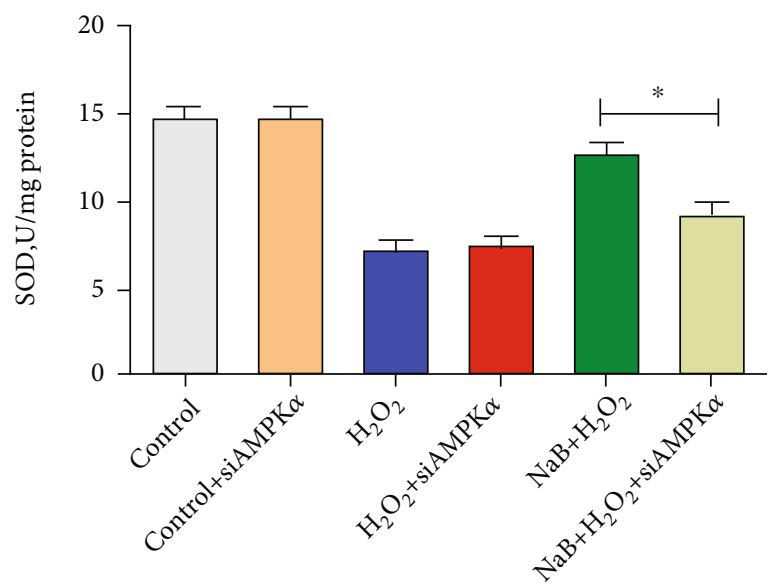

(b)

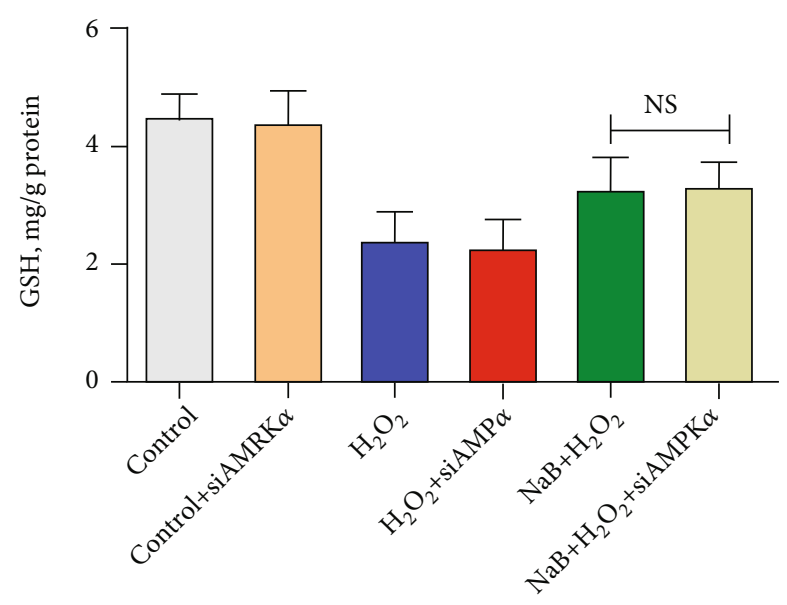

(c)

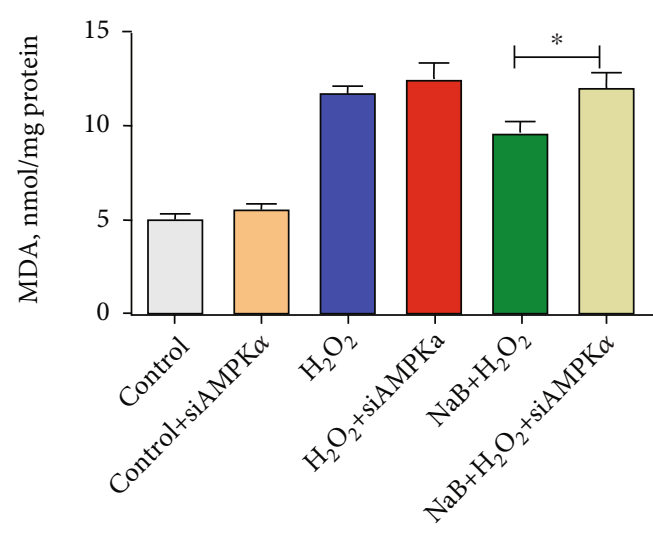

(d)

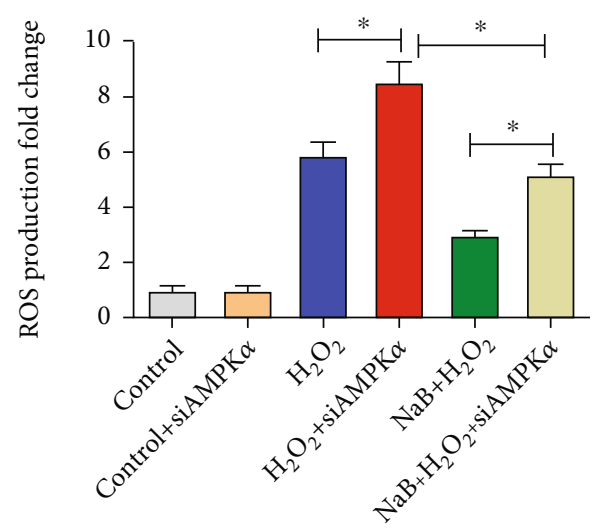

(e)

Figure 8: Continued. 


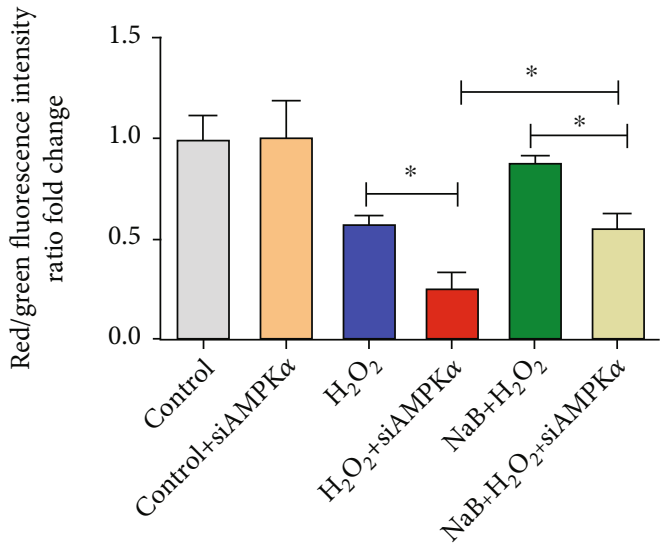

(f)
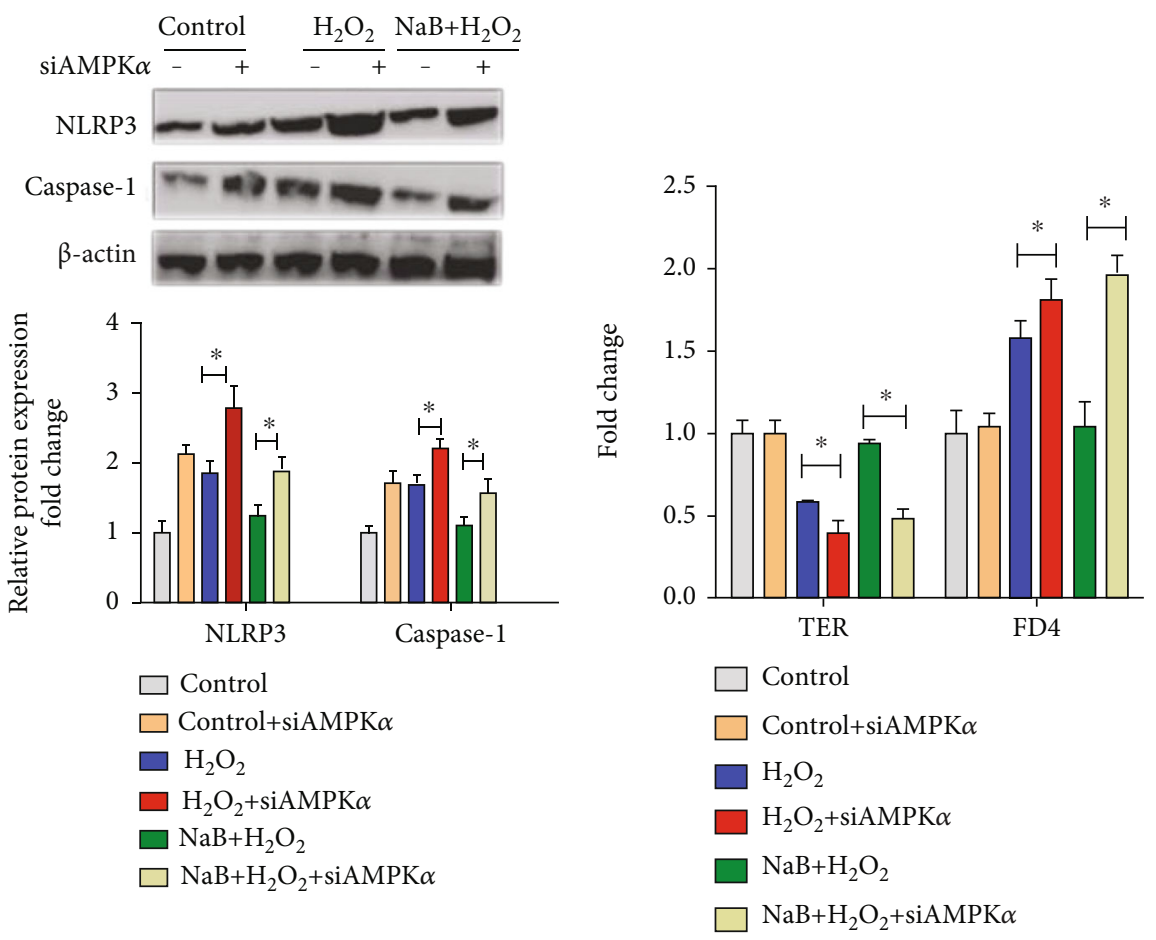

(g)

(h)

FIGURE 8: Effects of sodium butyrate ( $\mathrm{NaB}$ ) on mitophagy, oxidative stress, and intestinal epithelium barrier after interference with AMPK $\alpha$. (a) Expression and quantification of mitophagy proteins PINK1, Parkin, and P62. (b-d) Superoxide dismutase (SOD), glutathione reductase (GSH) activity, and malondialdehyde (MDA) content of porcine intestinal epithelial cells (IPEC-J2). (e) Cellular reactive oxygen species (ROS) level of IPEC-J2. (f) Cellular mitochondrial membrane potential of IPEC-J2. (g) Protein expression and quantification of recombinant NLR family, pyrin domain-containing protein 3 (NLRP3) and Caspase-1. (h) Intestinal epithelial transepithelial resistance (TER) and fluorescein isothiocyanate dextran $4 \mathrm{kDa}$ (FD4) permeability. * indicates a significant difference compared with the control group $(P<0.05)$.

and protein expression of NLRP3, Caspase-1, and IL-1 $\beta$ in IPEC-J2 cells and increased the content of IL- $1 \beta$, while sodium butyrate pretreatment alleviated this phenomenon. Similarly, NLRP3 inflammasome and IL-1 $\beta$ were activated by $\mathrm{H}_{2} \mathrm{O}_{2}$ in rat livers [37]. Jiang et al. also found that in bovine macrophages, sodium butyrate could reduce the inflammatory response caused by LPS by inhibiting the $\mathrm{NF}-\kappa \mathrm{B}$ and NLRP3 signaling pathways [38]. According to the results, we assumed that sodium butyrate could effec- tively inhibit the activation of NLRP3 and relieve intestinal inflammation induced by $\mathrm{H}_{2} \mathrm{O}_{2}$.

Mitophagy is a self-protection mechanism to remove dysfunctional mitochondria [39]. However, there were no reports about the effect of sodium butyrate on mitophagy of IPEC-J2 cells. We found that sodium butyrate enhanced mitophagy of IPEC-J2 cells, as indicated by increasing mRNA and protein level of mitophagy protein and promoting the colocalization of LC3B and mitochondria as 
well as the colocalization of PINK1, Parkin, and LC3B; meanwhile, we also found that sodium butyrate increased mitophagy vesicles under TEM. Similarly, Zhou et al. found that butyric acid can regulate intestinal epithelial cell autophagy through the HIF- $1 \alpha$ pathway to alleviate colitis [40]. Wang et al. found that sodium butyrate activated the PINK1-Parkin pathway and induced mitophagy [41]. J. S. Lee and G. M. Lee also found that sodium butyrate could induce mitophagy in Chinese hamster ovary $(\mathrm{CHO})$ cells, and mitophagy protein Parkin was recruited to mitochondria, suggesting that sodium butyrate induced mitophagy to remove damaged mitochondria [14]. According to the results above, it was reasonable to assume that sodium butyrate could regulate PINK1 and Parkin and trigger mitophagy to obliterate the damaged mitochondria caused by $\mathrm{H}_{2} \mathrm{O}_{2}$, which could prevent the intestine from metabolism disorders.

In the present experiment, we found that sodium butyrate protected IPEC-J2 cells from $\mathrm{H}_{2} \mathrm{O}_{2}$-induced oxidative damage, intestinal epithelium barrier damage, and mitochondrial impairment and enhanced mitophagy, but its specific molecular mechanism remained to be further studied. AMPK is a key energy sensor that can regulate cell energy metabolism [15]. Mollica et al. have reported that feeding sodium butyrate to insulin-resistant obese mice can increase liver AMPK activity, reduce ROS production, and improve liver mitochondrial function [20]. However, whether sodium butyrate could alleviate oxidative stress of IPEC-J2 cells through AMPK-dependent mitophagy remained to be further explored. Therefore, in the following experiments, we used AMPK inhibitor and mitophagy inhibitor to explore the specific role of AMPK signaling pathway and mitophagy in the protective effects of sodium butyrate on intestinal epithelium barrier. We found that sodium butyrate inhibited the decrease of SOD activity and the increase of MDA level induced by $\mathrm{H}_{2} \mathrm{O}_{2}$. However, the use of mitophagy inhibitor Mdivi-1 or AMPK inhibitor Compound C increased cellular MDA levels and reduced SOD activity. These results indicated that inhibition of mitophagy and AMPK could reduce the antioxidant effect of sodium butyrate. Similarly, Guo et al. found that butyric acid activated the AMPK signaling pathway through GPR109A in bovine mammary epithelial cells (BMECs) and then exerted an antioxidant effect [37]. We found that the ROS level and the percentage of depolarized cells significantly increased after using the mitophagy inhibitor Mdivi-1 or the AMPK inhibitor Compound C. Similarly, Zhao et al. reported that high concentrations of insulin in HepG2 cells could significantly reduce mitochondrial DNA and decrease the mitochondrial membrane potential, while sodium butyrate significantly increased the mitochondrial membrane potential and mitochondrial DNA, which was involved in the GPR43- $\beta$-AMPK signaling pathway [42]. We also previously reported that tributyrin activated the AMPK-mTOR signaling pathway and improved intestinal mitochondrial function in weaned piglets [25]. These results indicated that inhibition of mitophagy or AMPK $\alpha$ inhibited the protective effects of sodium butyrate on improving mitochondria under oxidative stress of IPEC-J2 cells. In addition, we found that the use of mitophagy inhibitor Mdivi-1 and AMPK inhibitor Compound $\mathrm{C}$ impaired intestinal epithelium barrier function, as indicated by the decreased TER and expression level of tight junction and the increased FD4 permeability of IPEC-J2 cells. Similarly, Elamin et al. reported that sodium butyrate alleviated the barrier dysfunction of Caco-2 cells induced by ethanol in vitro, of which the protective effects were suppressed by AMPK inhibitors or siRNA. Miao et al. found that in the Caco-2 cells, sodium butyrate activated AMPK, thereby promoting the reorganization of tight junctions [43]. Therefore, it was reasonable to assume that mitophagy and AMPK were necessary for sodium butyrate to improve the intestinal epithelium barrier under $\mathrm{H}_{2} \mathrm{O}_{2}$-induced oxidative stress. Moreover, we also found that the use of mitophagy inhibitor Mdivi-1 or AMPK inhibitor Compound C inhibited mitophagy, as indicated by decreasing expression level of mitophagy protein and the colocalization of PINK1 and LC3B, suggesting that inhibition of mitophagy or AMPK could suppress the mitophagy induced by sodium butyrate. In accordance with our results, Evans et al. found that butyrate induced autophagy-dependent cell apoptosis of human gingival epithelial cell Ca9-22 to alleviate periodontal disease, as indicated by activating AMPK and inducing the production of $\mathrm{LC} 3 \mathrm{~B}$, which was attenuated by AMPK inhibition; in addition, interference with LC3B gene could also significantly inhibit butyrate-induced cell death [44]. Hence, AMPK-mediated mitophagy is necessary for sodium butyrate's protective effects against oxidative stress.

In order to further confirm the role of AMPK in sodium butyrate-induced mitophagy of IPEC-J2 cells, we then used siRNA technology to knock down AMPK $\alpha$, which was known as the dominating AMPK catalytic subunit. We found that interference with AMPK $\alpha$ reduced the expression level of PINK1 and Parkin and increased the level of P62 when compared with the $\mathrm{H}_{2} \mathrm{O}_{2}$ group and the $\mathrm{NaB}+\mathrm{H}_{2} \mathrm{O}_{2}$ group. Similarly, Luo et al. found that sodium butyrate increased the mRNA and protein expression level of LC3B and activated phosphorylated AMPK, which was suppressed by treatment with siRNA AMPK in colorectal cancer cells [45]. These results indicated that AMPK $\alpha$ can mediate the mitophagy induced by sodium butyrate. Next, we found that interference with AMPK $\alpha$ significantly increased the level of MDA and ROS production and decreased MMP, indicating that the protective effects of sodium butyrate on alleviating cellular oxidative stress and mitochondrial dysfunction were weakened. In addition, interference with AMPK $\alpha$ significantly increased the protein expression of NLRP3 and Caspase-1 in IPEC-J2 cells, indicating that interference with AMPK $\alpha$ weakened the inhibition effect of sodium butyrate on NLRP3 inflammation factors. At the same time, interference with AMPK $\alpha$ impaired intestinal epithelium barrier function, as indicated by decreasing TER and increasing FD4 permeability of IPEC-J2 cells, suggesting that interference AMPK $\alpha$ weakened the protective effects of sodium butyrate on the intestinal epithelium barrier. Our results suggested that sodium butyrate could promote mitophagy via AMPK activation, protect mitochondria, and exert a protective effect on intestinal epithelium barrier of IPEC-J2 cells under oxidative stress. 


\section{Conclusion}

In conclusion, our work revealed that sodium butyrate ameliorated oxidative stress and inflammation and enhanced intestinal epithelium barrier function and mitochondrial function through AMPK-mitophagy pathway in IPEC-J2 cells.

\section{Abbreviations}

AMPK: Adenosine $5^{\prime}$-monophosphate- (AMP-) activated protein kinase

CC: $\quad$ Compound C

Cyt c: Cytochrome c

FD4: $\quad$ Fluorescein isothiocyanate dextran $4 \mathrm{kDa}$

GSH: Glutathione reductase

$\mathrm{H}_{2} \mathrm{O}_{2}$ : Hydrogen peroxide

IPEC-J2: Porcine intestinal epithelial cells

IL-1 $\beta$ : $\quad$ Interleukin- $1 \beta$

SOD: $\quad$ Superoxide dismutase

LC3B: Light-chain-associated protein B

mtDNA: Mitochondrial DNA

MDA: Malondialdehyde

Mdivi-1: Mitochondrial division inhibitor 1

NRF-1: Nuclear respiratory factor-1

NaB: $\quad$ Sodium butyrate

NLRP3: Recombinant NLR family, pyrin domaincontaining protein 3

PINK1: PTEN-induced putative kinase 1

PGC-1 $\alpha$ : Peroxisomal proliferator-activated receptor-g coactivator- $1 \alpha$

ROS: $\quad$ Reactive oxygen species

TFAM: Mitochondrial transcription factor A

TER: Transepithelial resistance

ZO-1: Zonula occludens-1.

\section{Data Availability}

The data used to support the findings of this study are available from the corresponding author upon logical request.

\section{Ethical Approval}

All experiments were carried out in accordance with the Chinese Guidelines for Animal Welfare and Experimental Protocol and were approved by the Animal Care and Use Committee of Zhejiang University.

\section{Conflicts of Interest}

The authors declare that there is no conflict of interest.

\section{Authors' Contributions}

Caihong $\mathrm{Hu}$ designed the whole scheme of the experiments; Xin Li, Chunchun Wang, Jiang Zhu, Qian Lin, Minjie Yu, and Jiashu Wen conducted the experiments; Xin Li and Chunchun Wang analyzed data and prepared the initial manuscript; Jie Feng reviewed the manuscript; Caihong $\mathrm{Hu}$ had primary responsibility for final content. All authors read and approved the final manuscript. Chunchun Wang is cofirst author.

\section{Acknowledgments}

This research was supported by Zhejiang Provincial Natural Science Foundation of China under Grant No. LZ20C170003, National Natural Science Foundation of China (32172740 and 31872387), and Zhejiang Provincial Key R\&D Program of China (2021C02008).

\section{Supplementary Materials}

Table 1: primer sequence for different genes of quantitative real-time (RT-qPCR) analysis. (Supplementary Materials)

\section{References}

[1] S. Cao, H. Xiao, X. Li et al., "AMPK-PINK1/Parkin mediated mitophagy is necessary for alleviating oxidative stressinduced intestinal epithelial barrier damage and mitochondrial energy metabolism Dysfunction in IPEC-J2," Antioxidants, vol. 10, no. 12, p. 2010, 2021.

[2] J. Yin, J. Duan, Z. Cui, W. Ren, T. Li, and Y. Yin, "Hydrogen peroxide-induced oxidative stress activates NF- $\kappa \mathrm{B}$ and Nrf2/ Keap1 signals and triggers autophagy in piglets," RSC Advances, vol. 5, no. 20, pp. 15479-15486, 2015.

[3] S. Cao, H. Wu, C. Wang et al., "Diquat-induced oxidative stress increases intestinal permeability, impairs mitochondrial function, and triggers mitophagy in piglets1," Journal of Animal Science, vol. 96, no. 5, pp. 1795-1805, 2018.

[4] M. Carocho and I. C. F. R. Ferreira, "A review on antioxidants, prooxidants and related controversy: natural and synthetic compounds, screening and analysis methodologies and future perspectives," Food and Chemical Toxicology, vol. 51, pp. 1525, 2013.

[5] C. Huang, P. Song, P. Fan, C. Hou, P. Thacker, and X. Ma, "Dietary sodium butyrate decreases postweaning diarrhea by modulating intestinal permeability and changing the bacterial communities in weaned piglets," The Journal of Nutrition, vol. 145, no. 12, pp. 2774-2780, 2015.

[6] D. S. Nielsen, B. B. Jensen, P. K. Theil, T. S. Nielsen, K. E. Knudsen, and S. Purup, "Effect of butyrate and fermentation products on epithelial integrity in a mucus-secreting human colon cell line," Journal of Functional Foods, vol. 40, pp. 917, 2018.

[7] E. Grilli, B. Tugnoli, C. J. Foerster, and A. Piva, "Butyrate modulates inflammatory cytokines and tight junctions components along the gut of weaned pigs," Journal of Animal Science, vol. 94, supplement 3, pp. 433-436, 2016.

[8] X. Ma, P. Fan, L. S. Li, S. Y. Qiao, G. L. Zhang, and D. F. Li, "Butyrate promotes the recovering of intestinal wound healing through its positive effect on the tight junctions1," Journal of Animal Science, vol. 90, supplement 4, pp. 266-268, 2012.

[9] E. E. Elamin, A. A. Masclee, J. Dekker, H. J. Pieters, and D. M. Jonkers, "Short-chain fatty acids activate AMP-activated protein kinase and ameliorate ethanol-induced intestinal barrier dysfunction in Caco-2 cell monolayers," The Journal of Nutrition, vol. 143, no. 12, pp. 1872-1881, 2013.

[10] R. Zhang, B. Liu, X. Fan et al., “Aldehyde dehydrogenase 2 protects against post-cardiac arrest myocardial dysfunction 
through a novel mechanism of suppressing mitochondrial reactive oxygen species production," Frontiers in Pharmacology, vol. 11, 2020.

[11] S. Cao, C. Wang, J. Yan, X. Li, J. Wen, and C. Hu, "Curcumin ameliorates oxidative stress-induced intestinal barrier injury and mitochondrial damage by promoting Parkin dependent mitophagy through AMPK-TFEB signal pathway," Free Radical Biology \& Medicine, vol. 147, pp. 8-22, 2020.

[12] M. Hytti, E. Korhonen, J. M. Hyttinen et al., "Antimycin Ainduced mitochondrial damage causes human RPE cell death despite activation of autophagy," Oxidative Medicine and Cellular Longevity, vol. 2019, Article ID 1583656, 12 pages, 2019.

[13] K. Wang and D. J. Klionsky, "Mitochondria removal by autophagy," Autophagy, vol. 7, no. 3, pp. 297-300, 2011.

[14] J. Seong Lee and G. M. Lee, "Effect of sodium butyrate on autophagy and apoptosis in Chinese hamster ovary cells," Biotechnology Progress, vol. 28, no. 2, pp. 349-357, 2012.

[15] D. G. Hardie, F. A. Ross, and S. A. Hawley, "AMPK: a nutrient and energy sensor that maintains energy homeostasis," Nature Reviews Molecular Cell Biology, vol. 13, no. 4, pp. 251-262, 2012.

[16] R. C. Laker, J. C. Drake, R. J. Wilson et al., “Ampk phosphorylation of Ulk1 is required for targeting of mitochondria to lysosomes in exercise-induced mitophagy," Nature Communications, vol. 8, no. 1, p. 548, 2017.

[17] E. Q. Toyama, S. Herzig, J. Courchet et al., "AMP-activated protein kinase mediates mitochondrial fission in response to energy stress," Science, vol. 351, no. 6270, pp. 275-281, 2016.

[18] S. Herzig and R. J. Shaw, "AMPK: guardian of metabolism and mitochondrial homeostasis," Nature Reviews Molecular Cell Biology, vol. 19, no. 2, pp. 121-135, 2018.

[19] D. R. Donohoe, N. Garge, X. Zhang et al., "The microbiome and butyrate regulate energy metabolism and autophagy in the mammalian colon," Cell Metabolism, vol. 13, no. 5, pp. 517-526, 2011.

[20] M. P. Mollica, G. M. Raso, G. Cavaliere et al., "Butyrate regulates liver mitochondrial function, efficiency, and dynamics in insulin-resistant obese mice," Diabetes, vol. 66, no. 5, pp. 1405-1418, 2017.

[21] J. R. Turner, B. K. Rill, S. L. Carlson et al., "Physiological regulation of epithelial tight junctions is associated with myosin light-chain phosphorylation," American Journal of Physiology-Cell Physiology, vol. 273, no. 4, pp. C1378-C1385, 1997.

[22] M. Chalubinski, K. Wojdan, P. Gorzelak, M. Borowiec, and M. Broncel, "The effect of oxidized cholesterol on barrier functions and IL-10 mRNA expression in human intestinal epithelium co-cultured with dendritic cells in the transwell system," Food and Chemical Toxicology, vol. 69, pp. 289-293, 2014.

[23] H. J. Shin, J. M. Han, Y. S. Choi, and H. J. Jung, "Pterostilbene suppresses both cancer cells and cancer stem-like cells in cervical cancer with superior bioavailability to resveratrol," Molecules, vol. 25, no. 1, p. 228, 2020.

[24] M. Z. Saleem, M. Alshwmi, H. Zhang et al., "Inhibition of JNKmediated autophagy promotes proscillaridin A- induced apoptosis via ROS generation, intracellular $\mathrm{Ca}\left({ }^{2+}\right)$ oscillation and inhibiting STAT3 signaling in breast cancer cells," Frontiers in Pharmacology, vol. 11, pp. 01055-01055, 2020.

[25] C. Wang, S. Cao, Z. Shen et al., "Effects of dietary tributyrin on intestinal mucosa development, mitochondrial function and AMPK-mTOR pathway in weaned pigs," Journal of Animal Science and Biotechnology, vol. 10, no. 1, 2019.
[26] C. Xue, Y. Li, H. Lv et al., "Oleanolic acid targets the gut-liver axis to alleviate metabolic disorders and hepatic steatosis," Journal of Agricultural and Food Chemistry, vol. 69, no. 28, pp. 7884-7897, 2021.

[27] I. Russo, A. Luciani, P. De Cicco, E. Troncone, and C. Ciacci, "Butyrate attenuates lipopolysaccharide-induced inflammation in intestinal cells and Crohn's mucosa through modulation of antioxidant defense machinery," PLoS One, vol. 7, no. 3, article e32841, 2012.

[28] H. P. Indo, M. Davidson, H. C. Yen et al., "Evidence of ROS generation by mitochondria in cells with impaired electron transport chain and mitochondrial DNA damage," Mitochondrion, vol. 7, no. 1-2, pp. 106-118, 2007.

[29] L. Li, H. H. Wang, X. T. Nie, W. R. Jiang, and Y. S. Zhang, "Sodium butyrate ameliorates lipopolysaccharide-induced cow mammary epithelial cells from oxidative stress damage and apoptosis," Journal of Cellular Biochemistry, vol. 120, no. 2, pp. 2370-2381, 2019.

[30] X. Xing, Z. Jiang, X. Tang et al., "Sodium butyrate protects against oxidative stress in HepG2 cells through modulating Nrf2 pathway and mitochondrial function," Journal of Physiology and Biochemistry, vol. 73, no. 3, pp. 405-414, 2016.

[31] D. Y. Liu, W. J. Lou, D. Y. Zhang, and S. Y. Sun, "ROS plays a role in the neonatal rat intestinal barrier damages induced by hyperoxia," BioMed Research International, vol. 2020, Article ID 8819195, 12 pages, 2020.

[32] X. Li, J. Wen, L. Jiao et al., "Dietary copper/zinc-loaded montmorillonite improved growth performance and intestinal barrier and changed gut microbiota in weaned piglets," Journal of Animal Physiology and Animal Nutrition, vol. 105, no. 4, pp. 678-686, 2021.

[33] M. C. Valenzano, K. Diguilio, J. Mercado et al., "Remodeling of tight junctions and enhancement of barrier integrity of the CACO-2 intestinal epithelial cell layer by micronutrients," PLoS One, vol. 10, no. 7, article e0133926, 2015.

[34] H. B. Wang, P. Y. Wang, X. Wang, Y. L. Wan, and Y. C. Liu, "Butyrate enhances intestinal epithelial barrier function via up-regulation of tight junction protein claudin-1 transcription," Digestive Diseases and Sciences, vol. 57, no. 12, pp. 3126-3135, 2012.

[35] H. Yan and K. M. Ajuwon, "Butyrate modifies intestinal barrier function in IPEC-J2 cells through a selective upregulation of tight junction proteins and activation of the Akt signaling pathway," PLoS One, vol. 12, no. 6, article e0179586, 2017.

[36] L. Peng, Z. R. Li, R. S. Green, I. R. Holzman, and J. Lin, "Butyrate enhances the intestinal barrier by facilitating tight junction assembly via activation of AMP-activated protein kinase in Caco-2 cell monolayers," The Journal of Nutrition, vol. 139, no. 9, pp. 1619-1625, 2009.

[37] W. Guo, J. Liu, J. Sun et al., "Butyrate alleviates oxidative stress by regulating NRF2 nuclear accumulation and H3K9/14 acetylation via GPR109A in bovine mammary epithelial cells and mammary glands," Free Radical Biology and Medicine, vol. 152, pp. 728-742, 2020.

[38] L. Jiang, J. Wang, Z. Liu et al., "Sodium butyrate alleviates lipopolysaccharide-induced inflammatory responses by down-regulation of NF- $\kappa \mathrm{B}$, NLRP3 signaling pathway, and activating histone acetylation in bovine macrophages," Frontiers in Veterinary Science, vol. 7, 2020.

[39] S. Saita, M. Shirane, and K. I. Nakayama, "Selective escape of proteins from the mitochondria during mitophagy," Nature Communications, vol. 4, no. 1, p. 1410, 2013. 
[40] C. Zhou, L. Li, T. Li et al., "SCFAs induce autophagy in intestinal epithelial cells and relieve colitis by stabilizing HIF-1 $\alpha$," Journal of Molecular Medicine, vol. 98, no. 8, pp. 1189-1202, 2020.

[41] F. Wang, H. Wu, M. Fan et al., "Sodium butyrate inhibits migration and induces AMPK-mTOR pathway-dependent autophagy and ROS-mediated apoptosis via the miR-139-5p/ Bmi-1 axis in human bladder cancer cells," The FASEB Journal, vol. 34, no. 3, pp. 4266-4282, 2020.

[42] T. Zhao, J. Gu, H. Zhang et al., "Sodium butyrate-modulated mitochondrial function in high-insulin induced HepG2 cell dysfunction," Oxidative Medicine and Cellular Longevity, vol. 2020, Article ID 579674, 16 pages, 2020.

[43] W. Miao, X. Wu, K. Wang et al., "Sodium butyrate promotes reassembly of tight junctions in Caco-2 monolayers involving inhibition of MLCK/MLC2 pathway and phosphorylation of PKC $\beta 2$," International Journal of Molecular Sciences, vol. 17, no. 10, p. 1696, 2016.

[44] M. Evans, T. Murofushi, H. Tsuda et al., "Combined effects of starvation and butyrate on autophagy-dependent gingival epithelial cell death," Journal of Periodontal Research, vol. 52, no. 3, pp. 522-531, 2017.

[45] S. Luo, Z. Li, L. Mao, S. Chen, and S. Sun, "Sodium butyrate induces autophagy in colorectal cancer cells through LKB1/ AMPK signaling," Journal of Physiology and Biochemistry, vol. 75, no. 1, pp. 53-63, 2019. 